\title{
Predictive Value of The G1-G6 Transcriptomic Molecular Classification of Hepatocellular Carcinoma for its Biological Behavior and Clinicopathological Features in China
}

\section{Ruonan Chen}

Fujian Medical University School of Basic Medical Sciences

\section{Zixiong Zhou}

Fujian Medical University School of Basic Medical Sciences

\section{Wubing $\mathrm{He}$}

Fujian Medical University

\section{Yi Chen}

Mengchao Hepatobiliary Hospital of Fujian Medical University

\section{Aimin Huang}

Fujian Medical University School of Basic Medical Sciences

Lihong Chen ( $\nabla$ drlhchen@sina.com )

Fujian Medical University School of Basic Medical Sciences

\section{Research}

Keywords: Hepatocellular carcinoma, G1-G6 molecular classification, China

Posted Date: October 12th, 2021

DOI: https://doi.org/10.21203/rs.3.rs-956345/v1

License: (c) (i) This work is licensed under a Creative Commons Attribution 4.0 International License.

Read Full License 


\section{Abstract}

Background: Hepatocellular carcinoma (HCC) is one of the most common cancers worldwide and a large number of genetic alterations are involved in the carcinogenetic process. A G1-G6 transcriptomic classification was previously proposed in a French study, and Korean and Singaporean groups indicated its potential application in Asian HCC patients. However, the genomic profiles of Chinese patients are distinct from patients of other regions, and therefore the suitability of this method in Chinese HCC patients has remained unknown.

Materials/Methods: In this study, we tested the transcriptomic group classification from the French cohort on a cohort of HCC patients from China. a total of 107 HCC cases from China were selected for the G1G6 transcriptomic molecular classification. The correlation between the G1-G6 molecular classification and clinicopathological features were analyzed. RNA sequencing and bioinformatics analysis were performed to screen related targets and molecular signaling pathways.

Results: We investigated the G1-G6 signatures in 107 Chinese HCC patients. HCC cases from China ( $n=107)$ were distributed as follows: G1 (17.76\%), G2 (1.87\%), G3 (18.69\%), G4 (9.35\%), G5 (23.36\%), and G6 (28.97\%) groups. We observed concordance between the genetic profiles and clinical features of Chinese HCC patients and French HCC patients. We found that the G1-G3 subgroups were associated with high serum alpha-fetoprotein (AFP) level, high copy number of hepatitis B virus (HBV) DNA, complex histopathological structure, macrovascular invasion, negative or weak Hep-Par1 expression, programmed death-ligand 1 expression, and liver cancer stemness. The G1 subgroup was mainly related to liver cancer stemness, and G3 subgroup showed the worst prognosis. The G5 and G6 subgroups were associated with activation of the Wnt/ $\beta$-catenin pathway. Compared with the G1-G4 group, the G1-G3 group showed significantly higher expression levels of regenerating family member 1 beta (REG1B), regenerating family member 3 gamma (REG3G), and inositol 1,4,5-trisphosphate receptor type 1 (ITPR1), and enriched calcium signaling pathway.

Conclusions: Our results clarify the correlation between G1-G6 molecular classification and molecular markers and molecular signaling pathways in the Chinese HCC population and initially established a link between the phenotype and molecular characteristics. This study enhances our understanding of the heterogenicity of China $\mathrm{HCC}$ and indicates that the $\mathrm{G} 1-\mathrm{G} 6$ signatures can be used to identify potential therapeutic biomarkers against HCC patients in China.

\section{Background}

Hepatocellular carcinoma (HCC) is the most common malignancy worldwide, with more than half of the new HCC cases and deaths every year occurring in China[1]. The main causes of HCC include chronic hepatitis B virus (HBV) or hepatitis C virus (HCV) infection, alcoholic or non-alcoholic steatohepatitis, autoimmune hepatitis, and several metabolic diseases. However, the etiologies of HCC between the EuroAmerican area and Asia-Pacific region vary widely. HBV infection was endemic in China, where the 
prevalence rate of HBsAg is 7.2\%[2], while HCV infection is common in western countries. In China, HCC only has a 5 -year survival rate of $14.1 \%$ and a recurrence rate of about $70 \%$ [3]. Approximately $70-80 \%$ of HCC patients are diagnosed at an advanced stage and can receive only palliative care[4]. Although several promising drugs were developed over the last decades, these drugs have failed to meet clinical endpoints in phase III trials[5]. The failure of these trials is, at least in part, because of the lack of effective molecular markers or the minimal validation of known molecular markers in different populations.

Recently, researchers have been attempting to establish a molecular classification for HCC, which is prognostically informative, to accurately identify patients after curative resection who will benefit from additional early therapeutic interventions to prevent a recurrence[6,7]. Several studies from China focused on the correlation between molecular markers and the differentiation degree and invasion ability of HCC. However, few studies have investigated the correlation between HCC molecular classification and pathological characteristics. A HCC classification method based on the expression of 16 genes was proposed by Boyault et al[8]. The authors classified tumors into six groups, G1-G6, using a minimal subset of 16 genes. This transcriptomic group classification was tested on cohorts from Singapore[9] and South Korea[10] with comparisons to European HCC patients. The results from these groups were generally in line with the results of Boyault et al, but there were still many inconsistencies. The Singapore study reported that the G1 subgroup had a higher proportion of patients with HBV in Singapore compared with European samples. HBV infection was found in the G2 subgroup of European HCC patients but not found in the G2 subgroup of Singapore HCC patients. In addition, the G6 subgroup was closely associated with satellite nodules in the European HCC population but not in the Singapore HCC population. The Singapore study also found no associations of clinical features with G4-G6 subgroups in the Southeast Asia HCC population. The probability of microvascular invasion (MVI) in the G3 subgroup was 5 times higher than other subgroups in Singapore samples. These differences suggested that the G1-G6 transcriptomic classification is not completely applicable to HCC patients in all regions of Asia. Furthermore, the genomic profiles of HCC patients are distinct in populations from China and other countries. For example, the TP53 mutation rate in Chinese populations is significantly lower than that in Korean and Singapore populations[11,12]. Therefore, it is valuable to validate the clinical relevance of the 16 gene HCC classification method in a Chinese population with HCC.

In this study, to determine whether the HCC molecular typing method established by the European team applies to Chinese HCC patients, a total of $107 \mathrm{HCC}$ cases from China were selected for the G1-G6 transcriptomic molecular classification. The correlation between the G1-G6 molecular classification and pathological features, biological behaviors, serum marker levels, 3-year overall survival (OS) rate, and other indicators were analyzed in combination with the clinicopathological data of the HCC patients. We also detected and analyzed molecular markers and molecular signal pathways related to the G1-G6 molecular classification. RNA sequencing and bioinformatics analysis were performed to screen related targets and molecular signaling pathways.

\section{Materials And Methods}




\section{HCC samples and clinical data}

The use of human clinical samples was approved by the Medical Ethics Committee of Mengchao Hepatobiliary Hospital of Fujian Medical University. A total of $120 \mathrm{HCC}$ specimens and paired adjacent noncancerous liver tissues were randomly selected from patients undergoing hepatectomy between January 2014 to December 2017. The patient follow-up information and clinicopathological characteristics were obtained from the biological sample bank and original pathology report. We also collected 12 fresh HCC tissues and paired adjacent noncancerous liver tissues for further analysis by RNA-seq. Data from The Cancer Genome Atlas (TCGA) database were obtained from TCGA portal sites (https://tcga-data.nci.nih.gov/tcga/tcgaDownload.jsp).

\section{Quantitative real-time PCR (qPCR)}

The qPCR procedures were performed as previously described[13] using Bestar ${ }^{\text {TM }}$ SYBR Green qPCR MasterMix kit (DBI-2043, DBI Bioscience, Shanghai, China). Briefly, total RNA was isolated from tissues using the TransZol Up Plus RNA Kit (ER501-01, TransGen Biotech, Beijing, China) according to the manufacturer's instructions. RNA quality was verified by ND-2000 (Nanodrop Products, Wilmington, DE, USA ), with only high-quality RNA samples (OD260/280 $=1.8 \sim 2.2$, OD260/230 $\geq 2.0$, RIN $\geq 8,28 S: 18 S \geq$

1.0) used for analyses. The RNA was converted to complementary DNA by reverse transcriptase using the Transcriptor First Strand cDNA Synthesis Kit (Roche, Basel, Switzerland) and qPCR analysis was performed. Specificity was verified by melting curve analysis. We used $18 \mathrm{~S}$ was used as an internal control, and gene expression analysis was performed using Applied Biosystems StepOne version 2.3 software. The primers are listed in Table 1. 
Table 1

Primer sequence of $\mathrm{qPCR}$

\begin{tabular}{|c|c|}
\hline Gene symbol & Sequence \\
\hline AFP-F & 5'-CTTTGGGCTGCTCGCTATGA-3' \\
\hline AFP-R & 5'-GCATGTTGATTTAACAAGCTGCT-3' \\
\hline $\mathrm{CDH} 2-\mathrm{F}$ & 5'-CGATAAGGATCAACCCCATACA-3' \\
\hline $\mathrm{CDH} 2-\mathrm{R}$ & 5'-TTCAAAGTCGATTGGTTTGACC-3' \\
\hline CYP2C9-F & 5'-ACATCAAGATTTTGAGCAGCC-3' \\
\hline CYP2C9-R & 5'-GGTTGGTTGTGCTTTTCCTTCT-3' \\
\hline EPHA1-F & 5'-CATACGCATGAAACGCTACATC-3' \\
\hline EPHA1-R & 5'-TCCTTGAATCCCTGAATACTGC-3' \\
\hline G0S2-F & 5'-CACTAAGGTCATTCCCGCCTCC-3' \\
\hline G0S2-R & 5'-ACAGTCTCCATCAGGCCGAG-3' \\
\hline HAMP-F & 5'-СTCTGTTTTCCCACAACAGAC-3' \\
\hline HAMP-R & 5'-CATCCCACACTTTGATCGATGA-3' \\
\hline HN1/JTP1-F & 5'-GCCTTTCTGGCCTCATGCTG-3' \\
\hline HN1/JTP1-R & 5'-GCCTGAAGTGTTTTGCTTTAGGT-3' \\
\hline LAMA3-F & 5'-CACCGGGATATTTCGGGAATC-3' \\
\hline LAMA3-R & 5'-AGCTGTCGCAATCATCACATT-3' \\
\hline MERTK-F & 5'-GAAGTTCATGGTGGATATTGCC-3' \\
\hline MERTK-R & 5'-CAGTTTCGAGCAGCTAAATCTC-3' \\
\hline NRAS-F & 5'-ATGACTGAGTACAAACTGGTGGT-3' \\
\hline NRAS-R & 5'-CATGTATTGGTCTCTCATGGCAC-3' \\
\hline PAK2-F & 5'-CACCCGCAGTAGTGACAGAG-3' \\
\hline PAK2-R & 5'-GGGTCAATTACAGACCGTGTG-3' \\
\hline PAP/REG3A-R & 5'-TGCAGACATAGGGTAACCTCACATT-3' \\
\hline PAP/REG3A-F & 5'-GGAGTGGAGTAGCAGTGATGT-3' \\
\hline PIR-F & 5'-AAAATGAACCCAGGAGATTTGC-3' \\
\hline PIR-R & 5'-ACACCATCCTTACTGGGTTTAG-3' \\
\hline RAB1A-F & 5'-AGATTAAAAAGCGAATGGGTCCC-3' \\
\hline
\end{tabular}




\begin{tabular}{|c|c|}
\hline Gene symbol & Sequence \\
\hline RAB1A-R & 5'-GCTTGACTGGAGTGCTCTGAAT-3' \\
\hline RAMP3-F & 5'-GTATAACGATCAGCGGGATGAG-3' \\
\hline RAMP3-R & 5'-TATGAGAGTTTCACCAACTGCA-3' \\
\hline SAE1-F & 5'-TGGGTCTGTTGGCCGAAATAG-3' \\
\hline SAE1-R & 5'-ACACAGCATCGAATTGAGTGAAA-3' \\
\hline EpCAM-F & 5'-GTCTGTGAAAACTACAAGCTGG-3' \\
\hline EpCAM-R & 5'-CAGTATTTTGTGCACCAACTGA-3' \\
\hline GS-F & 5'-CCGTGCCTATGATCCCAAGG-3' \\
\hline GS-R & 5'-CGCTACGATTGGCTACACCA-3' \\
\hline SOX9-F & 5'-GACAGCCCCCTATCGACTTC-3' \\
\hline SOX9-R & 5'-CAAACTCGTTGACATCGAAGG-3' \\
\hline 18S rRNA-F & 5'-AGAAACGGCTACCACATCCA-3' \\
\hline 18S rRNA-R & 5'-CACCAGACTTGCCCTCCA-3' \\
\hline
\end{tabular}

\section{G1-G6 molecular classification of HCC tumors}

The expression of 16 predictor genes (AFP, CDH2, CYP2C9, EPHA1, GOS2, HAMP, HN1/JPT1, LAMA3, MERTK, NRAS, PAK2, PAP/REG3A, PIR, RAB1A, RAMP3, and SAE1) in HCC patients was detected by qPCR following the methods described above. The gene expressions were analyzed using the mathematical model constructed by Boyault et al[8]. The tumors were then classified into G1-G6 transcriptomic subgroups.

\section{Western blot}

Tissues were harvested and lysed in cold extraction buffer (RIPA, Beyotime Biotechnology, Shanghai, China) as previously described[14]. Samples were centrifuged at $13,000 \times \mathrm{g}$ at $4^{\circ} \mathrm{C}$ for $15 \mathrm{~min}$, and the protein concentration in the supernatants was measured using the Pierce BCA Protein Assay kit (Thermo Fisher Scientific Inc., Waltham, IL, USA) according to the manufacturer's protocol. Equal amounts of protein samples were subjected to $10 \%$ sodium dodecyl sulfate-polyacrylamide gel electrophoresis and subsequently electro-transferred to polyvinylidene difluoride membranes. The membranes were blocked with $5 \%$ BSA diluted in Tris-buffered saline (20 mM Tris, $150 \mathrm{mM} \mathrm{NaCl}, \mathrm{pH}$ 7.4) containing $0.05 \%$ Tween20 for $2 \mathrm{~h}$ and then incubated with primary antibodies at $4^{\circ} \mathrm{C}$ overnight. The primary antibodies are listed in Table 2. The membranes were washed five times and then incubated with peroxidase-conjugated secondary antibodies (1:3,000, ab6721, Abcam, Cambridge, MA, USA) in blocking buffer for $1 \mathrm{~h}$ at RT. Band intensities were quantitated by an enhanced chemiluminescence detection system using the 
SuperSignal ${ }^{\text {TM }}$ West Pico Plus Kit (Thermo Fisher Scientific Inc.). The protein density was quantified with Bio-Rad Image Lab software and ImageJ software.

Table 2

Antibodies used for Western blot or IHC

\begin{tabular}{|lllll|}
\hline Antibodies & Host & Dilution (WB) & Dilution (IHC) & Manufacturer \\
\hline Primary Antibodies & & & & \\
\hline$\beta$-catenin & Rabbit & $1: 1000$ & $1: 300$ & CST \\
\hline EpCAM & Rabbit & $1: 1000$ & CST \\
GAPDH & Rabbit & $1: 3000$ & & Abcam \\
\hline GS & Rabbit & $1: 2000$ & & CST \\
PD-L1 & Rabbit & & $1: 1000$ & Abcam \\
SOX9 & Rabbit & & $1: 300$ & CST \\
\hline Secondary Antibodies & Goat & $1: 3000$ & & Abcam \\
\hline & Goat & & $1: 1$ & Maixin Inc. \\
\hline
\end{tabular}

Tissue microarray (TMA) construction

A total of 58 paraffin-embedded tissue samples from HCC patients (G1: 14 cases, G2: 1 case, G3: 14 cases, G4: 9 cases, G5: 10 cases, and G6: 10 cases) were randomly selected under a stratified sampling procedure to generate TMAs. The donor wax blocks were made of the paraffin sections and stained by hematoxylin-eosin (HE) staining. To exclude tissues with necrotic or bleeding areas, the corresponding positions of cancer tissues and adjacent liver tissues were observed and marked under a microscope.

The recipient block was cast by melting conventional paraffin wax in molds for making blank blocks. The donor tissue blocks were transferred into the recipient wax wells and prepared with a $1.5 \mathrm{~mm}$ perforated needle. Next, we placed the wax blocks in the oven at $65^{\circ} \mathrm{C}$ for approximately $7-9 \mathrm{~min}$ and removed them at the semi-melted state; the blocks were cooled slightly at room temperature and moved to a refrigerator at $4^{\circ} \mathrm{C}$. The freeze-thaw process was repeated so that the tissue core in the blocks and the wall of the pore were integrated.

\section{Immunohistochemistry (IHC) assay}

IHC staining was performed on liver sections using antibodies against $\beta$-catenin (1:300, \#8480, CST, Shanghai, China), EpCAM (1:1000, \#2929, CST), programmed death ligand 1 (PD-L1) (1:1000, ab228415, Abcam), and SOX9 (1:300, \#82630, CST). Briefly, the slides were deparaffinized and rehydrated, and antigen retrieval was performed using a high-temperature and high-pressure antigen repairing method. The sections were treated with a $3 \%$ peroxidase solution to block endogenous peroxidase. The sections were then incubated with $5 \%$ BSA blocking solution to reduce the non-specific background signal and false positives. Next, the sections were incubated overnight with the primary antibodies at $4^{\circ} \mathrm{C}$. A section 
was incubated in antibody diluent alone without the primary antibody as a control. The sections were further incubated with ImmPress horseradish peroxidase anti-rabbit IgG antibody (Maixin Inc., Fujian, China) secondary antibodies. The immunoreactions were visualized using a DAB Kit (Maixin Inc.). Following counterstaining and mounting, digital images from the sections were created using a wholeslide scanner (KF-PRO-005-EX, KFBIO, Ningbo, China) and images were captured and analyzed using KViewer version 1.5.3.1 software (KFBIO).

\section{RNA-seq and bioinformatics analysis}

Total RNA was extracted from tissues using Trizol reagent (TransGen Biotech) according to the manufacturer's instructions. RNA quality was confirmed using an Agilent 2100 Bioanalyzer. Libraries for sequencing were created with the Illumina NEBNext ${ }^{\circledR}$ Ultra $^{\text {TM }}$ RNA Library Prep Kit or NEBNext® Ultra ${ }^{\text {TM }}$ Directional RNA Library Prep Kit. Briefly, PCR amplification was performed to obtain the final DNA library. After the library was constructed, a Qubit2.0 Fluorometer was used for the preliminary quantification. RNA-seq was performed on the Illumina sequencing platform by Shanghai Jikai Company (Shanghai, China). Quality control indicated that the sequencing error rates and data filtering reads of the 24 samples were controlled within the acceptable range. RNA-seq, bioinformatics analysis, and TCGA database analysis were used to explore the target genes and molecular signaling pathways between the proliferation class (G1-G3, group H) and non-proliferation class (G4-G6, group L). We compared two or multiple gene expressions under different conditions using statistical methods, identified the specific genes that correlated with the conditions, and analyzed the biological significance (quality control, matching, quantitative analysis process, significant difference analysis, and function of enrichment) of these specific genes.

\section{Statistical analysis}

Statistical analysis was conducted by GraphPad Prism version 8 software (GraphPad Software, San Diego, CA, USA) and IBM SPSS Version 25 software. The chi-square test (Fisher exact probability method) was used to analyze the correlation between the G1-G6 molecular classification and tumor biological behavior. After checking data for normal distribution and variance homogeneity, comparisons between two groups were evaluated using independent-sample t-tests or Mann-Whitney $U$ test. For three or more groups, differences were statistically analyzed by one-way ANOVA or the non-parametric test (KruskalWallis test). Kaplan-Meier and Cox proportional hazards survival regression analysis were used to evaluate the prognostic significance of the G1-G6 molecular classifications within 36 months. All $p$ values were two-tailed, and $P<0.05$ was considered statistically significant.

\section{Results}

\section{Distribution of G1-G6 subgroups in HCC patients from Europe and China studies}


Gene expression analysis was performed on surgically resected HCC samples from Chinese patients who were grouped into $\mathrm{G} 1-\mathrm{G} 6$ transcriptomic categories according to the expression of 16 predictor genes. First, we isolated RNA samples from $120 \mathrm{HCC}$ tumor samples for HCC classification, and 107 RNA samples with sufficient tissue quantity and good quality were analyzed by qPCR. Data from these samples were analyzed according to the standard method proposed by the Europe group[8]. We found that the HCC cases from China ( $n=107)$ were distributed as follows: $G 1: n=19(17.76 \%), G 2: n=2(1.87 \%)$, G3: $n=20$ (18.69\%), G4: $n=10$ (9.35\%), G5: n=25 (23.36\%), and G6: $n=31$ (28.97\%) subgroups.

Corresponding counts for European patients[8] ( $n=120)$ were G1: $n=11$ (9.2\%), G2: $n=17(14.2 \%), G 3: n=15$ (12.5\%), G4: $n=41$ (34.2\%), G5: $n=24$ (20.0\%), and G6: $n=12$ (10.0\%) (Fig. 1A). Statistical differences between the groups were detected by Fisher exact test $(P<0.0001)$.

Several studies have shown that no matter what molecular typing method is used, HCC can be defined into two major groups, including a proliferation group and a non-proliferation group. Proliferation tumors are associated with aberrant activation of signaling pathways, while non-proliferative tumors display a well-differentiated phenotype[15,16]. Correspondingly, G1-G3 (combined with G1, G2, and G3) of the G1G6 molecular typing can be classified as a proliferation group, while G4-G6 (combined with G4, G5, and G6) was classified as a non-proliferative group. Next, we pooled classes into the G1-G3 group and G4G6 group. We found that the proportions of the G1-G3 group and $\mathrm{G} 4-\mathrm{G} 6$ group in the China cohort were $38.32 \%$ and $61.68 \%$, respectively. In the European cohort, $35.83 \%$ of HCC tissue samples were classified as $\mathrm{G} 1-\mathrm{G} 3$ and $64.17 \%$ as $\mathrm{G} 4-\mathrm{G} 6$. The ratio of $\mathrm{G} 1-\mathrm{G} 3 / \mathrm{G} 4-\mathrm{G} 6$ for China versus Europe did not show a statistical difference as determined by Fisher exact probability method ( $P=0.783$, Fig. 1B).

\section{Association of G1-G6 transcriptomic groups with HCC clinical variables}

We next summarized the characteristics and features of Chinese HCC patients according to G1-G6 transcriptomic classification (Table 3-1). In the Chinese HCC cohort, G1-G6 molecular classification was significantly correlated with tumor number $(P=0.035)$, serum AFP level $(P=0.009)$, HBV DNA copy number $(P=0.004)$, cirrhosis $(P=0.039)$, and MVI $(P=0.028)$. Additionally, the $\mathrm{G} 1$ subgroup was associated with high serum AFP level $(P=0.001)$. The complex histological subtype $(P=0.021)$ and high copy number of HBV DNA $(P=0.014)$ were found in the $\mathrm{G} 3$ subgroup. The $\mathrm{G} 4$ subgroup was correlated with advanced age $(P=0.012)$ and low copy number of HBV DNA $(P=0.012)$. The $\mathrm{G} 5$ subgroup was characterized by a single tumor $(P=0.013)$ and low incidence of MVI $(P=0.007)$. The $\mathrm{G} 6$ subgroup was associated with strong positive Hep-Par1 $(P=0.007)$ (Table 3-2).

Table 3-1. The characteristics and features of 107 Chinese HCC patients 


\begin{tabular}{|c|c|c|c|c|c|c|c|c|}
\hline \multirow[t]{2}{*}{ Variables } & \multirow[t]{2}{*}{$\mathbf{n}$} & \multirow[t]{2}{*}{ Category } & \multicolumn{6}{|c|}{ Transcriptomic group } \\
\hline & & & G1 & G2 & G3 & G4 & G5 & G6 \\
\hline \multirow[t]{2}{*}{ Gender } & 107 & Male & 13 & 2 & 19 & 9 & 19 & 27 \\
\hline & & Female & 6 & 0 & 1 & 1 & 6 & 4 \\
\hline \multirow[t]{2}{*}{ Age (years) } & 107 & $>55$ & 10 & 1 & 9 & 9 & 12 & 15 \\
\hline & & $\leq 55$ & 9 & 1 & 11 & 1 & 13 & 16 \\
\hline \multirow[t]{2}{*}{ Tumor size (cm) } & 107 & $>5$ & 15 & 2 & 18 & 6 & 18 & 27 \\
\hline & & $\leq 5$ & 4 & 0 & 2 & 4 & 7 & 4 \\
\hline \multirow[t]{2}{*}{ Tumor number } & 107 & $>1$ & 5 & 0 & 4 & 3 & 0 & 3 \\
\hline & & 1 & 14 & 2 & 16 & 7 & 25 & 28 \\
\hline \multirow[t]{2}{*}{$\mathrm{AFP}(\mathrm{ng} / \mathrm{mL})$} & 94 & $>400$ & 12 & 0 & 7 & 2 & 5 & 9 \\
\hline & & $\leq 400$ & 4 & 1 & 8 & 8 & 17 & 21 \\
\hline \multirow[t]{2}{*}{ CEA (ng/mL) } & 102 & $>6.5$ & 3 & 0 & 2 & 0 & 0 & 3 \\
\hline & & $\leq 6.5$ & 14 & 2 & 17 & 10 & 24 & 27 \\
\hline \multirow[t]{2}{*}{ HBV DNA (IU/mL) } & 94 & $\geqq 500$ & 10 & 0 & 13 & 2 & 16 & 14 \\
\hline & & $<500$ & 6 & 1 & 2 & 8 & 6 & 16 \\
\hline \multirow[t]{2}{*}{ Tumor capsule } & 70 & Complete & 2 & 1 & 3 & 0 & 3 & 4 \\
\hline & & Incomplete & 10 & 0 & 12 & 8 & 11 & 16 \\
\hline \multirow[t]{2}{*}{ Cirrhosis } & 106 & Yes & 13 & 0 & 16 & 4 & 14 & 24 \\
\hline & & No & 5 & 2 & 4 & 6 & 11 & 7 \\
\hline \multirow[t]{2}{*}{ Histological subtype } & 106 & Trabecular & 11 & 0 & 7 & 8 & 18 & 20 \\
\hline & & Other & 8 & 2 & 12 & 2 & 7 & 11 \\
\hline \multirow[t]{2}{*}{ Macrovascular invasion } & 107 & Yes & 9 & 0 & 9 & 1 & 5 & 8 \\
\hline & & No & 10 & 2 & 11 & 9 & 20 & 23 \\
\hline \multirow[t]{2}{*}{ Microvascular invasion } & 107 & Positive & 15 & 0 & 16 & 8 & 12 & 24 \\
\hline & & Negative & 4 & 2 & 4 & 2 & 13 & 7 \\
\hline \multirow[t]{2}{*}{ Metastasis } & 107 & Yes & 0 & 0 & 1 & 2 & 1 & 1 \\
\hline & & No & 19 & 2 & 19 & 8 & 24 & 30 \\
\hline Satellite nodule & 107 & Positive & 9 & 0 & 9 & 4 & 10 & 12 \\
\hline
\end{tabular}




\begin{tabular}{|c|c|c|c|c|c|c|c|c|}
\hline & & Negative & 10 & 2 & 11 & 6 & 15 & 19 \\
\hline \multirow[t]{2}{*}{ Tumor differentiation } & 104 & I-II & 4 & 2 & 3 & 0 & 6 & 9 \\
\hline & & III-IV & 15 & 0 & 14 & 10 & 19 & 22 \\
\hline \multirow[t]{2}{*}{ TNM stage } & 107 & $\mathrm{I}-\mathrm{II}$ & 8 & 2 & 10 & 6 & 18 & 19 \\
\hline & & III-IV & 11 & 0 & 10 & 4 & 7 & 12 \\
\hline \multirow[t]{2}{*}{ BCLC stage } & 107 & $A-B$ & 10 & 2 & 11 & 7 & 18 & 21 \\
\hline & & C-D & 9 & 0 & 9 & 3 & 7 & 10 \\
\hline \multirow[t]{2}{*}{ Hep-Par1 } & 99 & $(-),(+),(++)$ & 16 & 2 & 14 & 9 & 18 & 18 \\
\hline & & $(+++),(++++)$ & 2 & 0 & 1 & 1 & 6 & 12 \\
\hline \multirow[t]{2}{*}{ GPC3 } & 100 & $(-),(+),(++)$ & 11 & 2 & 13 & 6 & 15 & 17 \\
\hline & & $(+++),(++++)$ & 7 & 0 & 3 & 4 & 9 & 13 \\
\hline \multirow[t]{2}{*}{ CK19 } & 101 & $(-),(+),(++)$ & 18 & 2 & 15 & 10 & 24 & 30 \\
\hline & & $(+++),(++++)$ & 0 & 0 & 1 & 0 & 1 & 0 \\
\hline
\end{tabular}

Table 3-2. Correlation analysis of clinicopathological features between G1-G6 types and HCC patients G1G6

\begin{tabular}{|c|c|c|c|}
\hline Variables & Transcriptomic group & $\mathbf{n}$ & $P$ value \\
\hline AFP (>400ng/mL) & G1 & 94 & $0.0009 * * *$ \\
\hline Histological subtype (complex) & G3 & 106 & $0.0207 *$ \\
\hline HBV DNA ( $\geq 500 \mathrm{IU} / \mathrm{mL})$ & G3 & 94 & $0.0135^{*}$ \\
\hline Age (>55 years) & G4 & 107 & $0.0120 *$ \\
\hline HBV DNA $(<500 \mathrm{IU} / \mathrm{mL})$ & G4 & 94 & $0.0115^{*}$ \\
\hline Tumor number (single) & G5 & 107 & $0.0132 *$ \\
\hline MVI (-) & G5 & 107 & $0.0072^{\star *}$ \\
\hline Hep-Par1(+++), (++++) & G6 & 99 & $0.0066 * *$ \\
\hline
\end{tabular}

To analyze the correlation between proliferation and clinicopathological characteristics of HCC patients based on G1-G6 molecular classification, we integrated the proliferative group (G1-G3) and nonproliferative group (G4-G6) by clinicopathological information. We found that the proliferative group (G1-G3) was correlated with high serum AFP level $(P=0.002)$, high copy number of HBV DNA $(P=0.047)$, complex histological subtype $(P=0.010)$, macrovascular invasion $(P=0.012)$, and negative or weak positive Hep-Par1 ( $P=0.012)$ (Table 4). 
Table 4. Correlation analysis between G1-G3 group HCC and clinicopathological characteristics of patients

\begin{tabular}{|c|c|c|c|c|c|}
\hline \multirow[t]{2}{*}{ Variables } & \multirow[t]{2}{*}{$\mathrm{n}$} & \multirow[t]{2}{*}{ Category } & \multicolumn{2}{|c|}{ Transcriptomic group } & \multirow[t]{2}{*}{$P$ value } \\
\hline & & & G1-G3 & G4-G6 & \\
\hline \multirow[t]{2}{*}{ AFP (ng/mL) } & 94 & $>400$ & 19 & 16 & $0.0016 * *$ \\
\hline & & $\leq 400$ & 13 & 46 & \\
\hline \multirow[t]{2}{*}{ HBV DNA (IU/mL) } & 94 & $\geqq 500$ & 23 & 32 & $0.0465^{\star}$ \\
\hline & & $<500$ & 9 & 30 & \\
\hline \multirow[t]{2}{*}{ Histological subtype } & 106 & Trabecular & 18 & 46 & $0.0104^{*}$ \\
\hline & & Other & 22 & 20 & \\
\hline \multirow[t]{2}{*}{ Macrovascular invasion } & 107 & Yes & 18 & 14 & $0.0119 *$ \\
\hline & & No & 23 & 52 & \\
\hline \multirow[t]{2}{*}{ Hep-Par1 } & 99 & $(-),(+),(++)$ & 32 & 45 & $0.0123 *$ \\
\hline & & $(+++),(++++)$ & 3 & 19 & \\
\hline
\end{tabular}

Using follow-up data of patients after hepatectomy, we also analyzed the correlation between HCC prognosis and G1-G6 molecular classification. As shown in Fig. 1C, the G1-G3 HCC patients showed a shorter 3-year OS than G4-G6 HCC patients $(P=0.010)$. The recurrence-free survival of the $\mathrm{G} 1-\mathrm{G} 3$ group tended to be shorter than the G4-G6 group ( $P=0.072$ ) (Figure 1D). Further analysis showed that $\mathrm{G} 1$ patients tended to have a shorter 3-year OS than non-G1 patients $(P=0.1867)$ (Figure 1E) and G3 patients had the worst prognosis $(P=0.0125)$ (Figure $1 \mathrm{~F})$. Cox regression univariate analysis showed that the TNM stage $(P=0.0002), \mathrm{BCLC}$ stage $(P=0.001)$, and $\mathrm{G} 1-\mathrm{G} 6$ molecular classification $(P=0.012)$ were important factors that affected the overall prognosis of HCC patients. All factors with a $p$-value $<0.1$ detected in univariate analyses were included in multivariate analyses, and the subsequent results showed that the G1-G6 molecular classification $(P=0.035)$ is an independent prognostic risk factor for poor OS of HCC patients (Table 5).

Table 5. Univariate and multivariate Cox regression analysis of overall survival rate of HCC patients 


\begin{tabular}{|c|c|c|c|c|c|}
\hline \multirow[t]{3}{*}{ Variables } & \multicolumn{5}{|c|}{ Survival } \\
\hline & \multicolumn{3}{|c|}{ Univariate analysis } & \multicolumn{2}{|c|}{ Multivariate analysis } \\
\hline & n & $\mathrm{HR}(95 \% \mathrm{Cl})$ & $P$ value & $\mathrm{HR}(95 \% \mathrm{Cl})$ & $P$ value \\
\hline $\begin{array}{l}\text { Gender (Female vs } \\
\text { Male) }\end{array}$ & 107 & $\begin{array}{l}0.754(0.318- \\
1.785)\end{array}$ & 0.520 & & \\
\hline Age (>55 vs $\leq 55 y r s)$ & 107 & $\begin{array}{l}1.577(0.868- \\
2.865)\end{array}$ & 0.135 & & \\
\hline $\begin{array}{l}\text { Tumor size (>5 vs } \\
\leq 5 \mathrm{~cm})\end{array}$ & 107 & $\begin{array}{l}0.886(0.412- \\
1.908)\end{array}$ & 0.758 & & \\
\hline $\begin{array}{l}\text { Tumor number (>1 vs } \\
\text { 1) }\end{array}$ & 107 & $\begin{array}{l}0.542(0.260- \\
1.129)\end{array}$ & 0.102 & & \\
\hline $\begin{array}{l}\text { Complete tumor } \\
\text { capsule } \\
\text { (Yes or No) }\end{array}$ & 70 & $\begin{array}{l}1.590(0.549- \\
4.602)\end{array}$ & 0.393 & & \\
\hline $\begin{array}{l}\text { AFP } \\
(>400 \text { vs } \leq 400 \mathrm{ng} / \mathrm{mL})\end{array}$ & 94 & $\begin{array}{l}0.883(0.272- \\
2.861)\end{array}$ & 0.835 & & \\
\hline HBV (Yes vs No) & 104 & $\begin{array}{l}1.041(0.409- \\
2.652)\end{array}$ & 0.933 & & \\
\hline Cirrhosis (Yes vs No) & 106 & $\begin{array}{l}0.684(0.352- \\
1.327)\end{array}$ & 0.261 & & \\
\hline $\begin{array}{l}\text { Tumor differentiation } \\
\text { (I-II vs III-IV) }\end{array}$ & 104 & $\begin{array}{l}0.590(0.273- \\
1.272)\end{array}$ & 0.178 & & \\
\hline $\begin{array}{l}\text { Microvascular } \\
\text { invasion } \\
\text { (Yes vs No) }\end{array}$ & 96 & $\begin{array}{l}0.613(0.309- \\
1.215)\end{array}$ & 0.161 & & \\
\hline $\begin{array}{l}\text { TNM stage } \\
\text { (I-II vs III-IV) }\end{array}$ & 107 & $\begin{array}{l}0.307(0.166- \\
0.568)\end{array}$ & $0.0002^{* \star *}$ & $\begin{array}{l}0.372(0.124- \\
1.117)\end{array}$ & 0.078 \\
\hline $\begin{array}{l}\text { BCLC stage } \\
(0-A \text { vs B-D) }\end{array}$ & 107 & $\begin{array}{l}0.347(0.191- \\
0.631)\end{array}$ & $0.001 * * *$ & $\begin{array}{l}0.868(0.299- \\
2.514)\end{array}$ & 0.794 \\
\hline $\begin{array}{l}\text { Molecular } \\
\text { classification } \\
\text { (G1-G3 vs G4-G6) }\end{array}$ & 107 & $\begin{array}{l}0.469(0.259- \\
0.847)\end{array}$ & $0.012^{*}$ & $\begin{array}{l}0.526(0.289- \\
0.956)\end{array}$ & $0.035^{*}$ \\
\hline
\end{tabular}

\section{Predictor of HCC classification using qPCR}


A total of 19 para-cancer liver tissue samples were randomly selected to investigate the correlation between expression of the 16 genes in the proliferation group (G1-G3) compared with the nonproliferation group (G4-G6). Our results showed that the expression levels of $A F P(P=0.0200), C D H 2$ $(P=0.0022), H N 1(P<0.0001), N R A S(P=0.0096), P A K 2(P=0.0024), R A B 1 A(P=0.0020)$, and SAE1 $(P<0.0001)$ were significantly higher in the proliferation group $(\mathrm{G} 1-\mathrm{G} 3)$ than in the non-proliferation group (G4-G6) (Fig. 2A). Conversely, the expression levels of $\angle A M A 3(P=0.0059)$ and $P A P(P=0.0495)$ were markedly higher in the non-proliferation group (G4-G6) than in the proliferation group (G1-G3) (Fig. 2B). There were no significant differences in the expressions of the following genes between the proliferation group (G1-G3) and non-proliferation group (G4-G6): CYP2C9 $(P=0.6426), E P H A 1(P=0.0531), G 0 S 2$ $(P=0.4714), \operatorname{HAMP}(P=0.0506), \operatorname{MERTK}(P=0.8628), P I R(P=0.9246)$, and RAMP3 ( $P=0.9190)$ (Fig. $2 C)$.

\section{PD-L1 is highly expressed in G1-G3 subgroups}

Several molecular targeted drugs have entered clinical trials as palliative and complementary treatments for HCC[17]. For example, the clinically approved antibodies targeting PD-1 or its ligand PD-L1 were shown to cause lasting responses in up to $25 \%$ of advanced HCC patients in two early trials. In HCC, the proportion of tumor cells expressing PD-L1 is approximately 15\%[18]. Several studies showed inflamed and membrane expression of PD-L1 in ancestral liver cancer HCC, while lymph epithelioma-like HCC is inflamed by inflammatory cells that express PD-L1 in large numbers[16,19,20]. The expression pattern of PD-L1 has an important effect on HCC prognosis, which is poor if both tumor cells and macrophages express PD-L1[21]. Screening of patients who may benefit from PD-1/PD-L1 inhibitor therapy is the most important clinical concern. The correlation between G1-G6 classification and tumor cell PD-L1 expression is not clear. Therefore, we examined the expression of the PD-L1 by IHC in a TMA.

The TMA comprised 58 resected HCC specimens and 58 specimens of adjacent non-malignant and premalignant para-cancer liver tissue from the same patients. Staining was compared on consecutive tissue sections of the TMA to enable automated (unbiased) image analysis and direct comparison of the samples. The staining was quantified according to the percentage of area stained for each protein in comparison with isotype-matched control IgG (Fig. 3A). Representative images for the positive PD-L1 antibody staining are shown in Fig. 3B, along with histograms showing the pooled quantified data for the antigen (Fig. $3 \mathrm{C}$ ). We found that the positive staining of PD-L1 in G1-G3 subgroups was significantly higher than in $\mathrm{G} 4-\mathrm{G} 6$ subgroups $(P=0.028)$.

\section{G5 and G6 HCC subgroups are associated with activation of the Wnt/ $\beta$-catenin pathway signaling}

Aberrant activation of Wnt/ $\boldsymbol{\beta}$-catenin signaling plays a key role in HCC progression, with approximately half of HCC cases acquiring mutations in either CTNNB1 or AXIN1[22]. Glutamine synthetase (GS) is classically overexpressed when the Wnt/ $\boldsymbol{\beta}$-catenin signaling pathway is activated due to CTNNB1 
mutations and could be used as surrogate marker[23]. In our study, we found that the protein levels of $\boldsymbol{\beta}$ catenin were substantially higher in G5 and G6 HCC subgroups than in G1-G4 subgroups (Fig. 4A and B). Additionally, the mRNA expression level of GS in HCC liver tissues was significantly increased compared with levels in para-cancer liver tissues, and the G5 and G6 HCC subgroups showed significantly higher expression levels of GS than the G1-G4 subgroups (Fig. 4C). In line with these results, western blot analysis showed that protein levels of $\boldsymbol{\beta}$-catenin and GS were higher in G5 and G6 subgroups than other subgroups (Fig. 4D). Further statistical analysis found that the ratio of GS expression to $\beta$-catenin expression in the G5 and G6 groups was approximately three-fold higher compared with levels in the G1G4 subgroups, which suggests that the Wnt/ $\beta$-catenin pathway is highly activated in G5 and G6 HCC. The activation level was highest in G6. These results suggested that G5 and G6 HCC subgroups were closely associated with the robust activation of $\mathrm{Wnt} / \boldsymbol{\beta}$-catenin signaling.

\section{The $\mathbf{G 1}$ subgroup is associated with maintenance of tumor cell stemness}

Increasing studies have reported the presence of a small number of cells in tumor tissue with strong stemness [24,25]. We found that the G1-G3 HCC subgroups were closely associated with poor differentiation and high invasion of tumor cells. We further checked whether the proliferation group (G1G3) was correlated with the stemness characteristics of HCC. Representative images for IHC of stemnessrelated molecular markers, EpCAM and Sox9, are shown in Fig. 5A and 5C. EpCAM staining was located at the cytomembrane and cytoplasm while SOX9 expression was identified in the nucleus. The positive expressions of EpCAM and Sox9 in the G1-G3 group were significantly higher than in the G4-G6 group (Fig. 5B and D). The G1 subgroup showed the highest positive percentages of EpCAM and Sox9 compared with other subgroups (Fig. 5E). The G3 subgroup did not show a significant difference compared with other subgroups (Fig. 5F). qPCR analysis also showed a similar pattern as the IHC results (Fig. $5 \mathrm{G}$ and $\mathrm{H}$ ). These results suggested that the $\mathrm{G} 1$ subgroup is associated with the maintenance of stemness in tumor cells.

\section{Transcriptome sequencing analysis for G1-G6 molecular classification of Chinese HCC patients}

The results of our study suggest that G1-G3 group can be used as an independent risk factor for evaluating HCC patients' prognosis and is significantly correlated with poor prognosis after hepatectomy. To clearly understand the poor prognosis group based on G1-G6 molecular classification of Chinese HCC patients, 12 samples of HCC tissues (2 cases of each subgroup) and the corresponding adjacent liver tissues were randomly selected. The expression levels of genes related to differentiation, proliferation, and function of tumor cells were examined in these samples and analyzed by RNA-seq and bioinformatics. The error rates of the 24 samples were all within the acceptable range (Supplementary Fig. 1). The proportions of filtered reads of the 24 samples were all within the acceptable range, and the 
proportion of clean reads of each sample accounted for more than $90 \%$, which suggested that the data were suitable for subsequent analysis (Supplementary Fig. 2).

The all HCC group (CA group) included 12 samples of HCC tissues, and the para-HCC group (pCA group) included 12 corresponding samples of adjacent liver tissues. High proliferative group (group H) includes 6 samples of G1-G3 cancer tissues, and low proliferative group (group L) includes 6 samples of G4-G6 cancer tissues. Compared with the pCA group, there were 3233 up-regulated genes and 1654 downregulated genes in the CA group, while 33,299 genes showed no changes between the two groups (Fig. 6A). Compared with group L (G4-G6 subgroup), there were 1,269 up-regulated and 1,445 down-regulated genes in group $\mathrm{H}$ (G1-G3 subgroup), and 33,692 genes showed no differential expression between the two groups (Fig. 6B). We also found that 108 genes overlapped between the $\mathrm{H}$ group (compared with group L) and CA (compared with group pCA) (Fig. 6C), and 27 genes were commonly down-regulated (Fig. 6D). Among the 27 genes, four genes (REG1B, REG3G, C19orf18, and ITPR1) were significantly upregulated or downregulated in group $\mathrm{H}$ compared with group $\mathrm{L}$ (Table 6). Analysis using TCGA database also confirmed that the expression levels of REG1B, REG3G, and ITPR1 were significantly increased in HCC liver tissues compared with normal liver tissues (Fig. 6E). Using the follow-up information database of TCGA patients, Kaplan-Meier analysis was conducted to analyze the prognosis of HCC patients. As shown in Fig. 6F, HCC patients with a high expression of ITPR1 showed a significantly shorter 3-year OS than HCC patients with low or medium expression of ITPR1. However, there were no statistical differences between $\mathrm{HCC}$ patients with high expressions of REG1B or REG3G and the corresponding control groups. These results suggested that ITPR1 may promote the occurrence and development of HCC and affect the prognosis of HCC patients.

\section{Table 6. Gene differential expression analysis}

\begin{tabular}{|lllll|}
\hline Gene name & Trendency & log2FoldChange & $P$ value & Padj \\
\hline REG1B & Up & 29.80 & $3.28 \mathrm{E}-23$ & $6.88 \mathrm{E}-19$ \\
\hline REG3G & Up & 25.10 & $6.34 \mathrm{E}-17$ & $6.65 \mathrm{E}-13$ \\
\hline C19orf18 & Down & 6.99 & $3.79 \mathrm{E}-07$ & $7.94 \mathrm{E}-04$ \\
\hline ITPR1 & Up & 2.67 & $7.46 \mathrm{E}-05$ & $2.50 \mathrm{E}-02$ \\
\hline
\end{tabular}

We next performed KEGG pathway analysis using the KEGG database and Clusterprofile software. Fisher exact analysis was used to test and calculate $p$-values, and the pathways with a $p$-value less than 0.05 were retained. The $p$-value represented the significance of enrichment of differential genes; a lower the $p$ value indicates a greater correlation between the pathway and the differential genes. A Padj value less than 0.05 was taken as the threshold of significant enrichment. The KEGG pathway enrichment analysis revealed that the calcium signaling pathway, cGMP-PKG signaling pathway, renin secretion, neuroactive ligand-receptor interaction, and cell metabolism-related signaling pathways were significantly enriched in the G1-G3 subgroups (Fig. 7A-B). 


\section{Discussions}

In this study, our results indicate that the G1-G6 signatures were generally in concordance between the genetic profiles of Chinese HCC patients and HCC patients in the original French study. Additionally, our data suggest that the G1-G3 group can be used as an independent risk factor for evaluating the prognosis of HCC patients, since the G1-G3 group was significantly correlated with poor prognosis after hepatectomy and the G3 group showed the worst prognosis among all subgroups. Furthermore, we demonstrated that the G5-G6 group was associated with activation of the Wnt/ $\beta$-catenin pathway. Moreover, we discovered that the G1-G3 group was associated with PD-L1 expression and the G1 subgroup is mainly related to liver cancer stemness. Importantly, we identified four genes (REG1B, REG3G, C19orf18, and ITPR1) that were significantly up-regulated or down-regulated in the proliferation group (G1-G3) compared with the non-proliferation group (G4-G6). Additionally, we found that the calcium signaling pathway, cGMP-PKG signaling pathway, renin secretion, neuroactive ligand-receptor interaction, and cell metabolism-related signaling pathways were significantly enriched in the G1-G3 subgroup.

Although our results were generally consistent with the studies from Europe and Singapore, there were still many inconsistencies. First, the distribution of the HCC subgroups in the Chinese cohort was significantly different from that in the original European cohort. In the European study, the G4 and G1 subgroups represented the largest and smallest subgroup, respectively. In our study, the G6 and G2 subgroups were the largest and smallest groups, respectively. Second, the Singapore research reported that the incidence of MVI in the G3 subgroup was markedly higher than in other subgroups. However, we did not find a similar characteristic of the G3 subgroup of China HCC patients. Instead, we found that the incidence of MVI was significantly lower in the G5 subgroup than the other subgroups. Third, inconsistent with the European study but in line with the Singapore study, we did not find any subgroups characterized with satellite nodules. Finally, our data showed that the G1-G3 subgroups had a shorter 3-year OS rate and the G3 subgroup has the worst prognosis, which was different from the studies in Europe and Singapore. These differences suggest that some of the clinical characteristics of HCC patients are distinct in the populations from China and other countries.

In this study, we found a high serum AFP level in the G1 group and a correlation between the G5-G6 group and Wnt/ $\beta$-catenin pathway activation based on previous studies. Several studies showed that HCC with $\boldsymbol{\beta}$-catenin mutations have slow proliferation, well-differentiated characteristics, and trabecular type false adenoid type histology, with cholestasis and intratumoral infiltrating immune deficiency[16,26]. $\beta$-catenin is a key molecular marker in the epithelial-mesenchymal transition. In physiological conditions, $\beta$-catenin binds cadherin and promotes the release of cadherin when $\beta$-catenin translocates to the nucleus[27]. In HCC, E-cadherin is reduced on the cell surface and N-cadherin is expressed once tumor cells undergo epithelial-mesenchymal transition[28]. It implicates that G5 and G6 subgroups may have some features as mentioned above.

We found several additional correlations in this study that had not been reported previously. For example, the G1-G3 group was associated with PD-L1 expression and liver cancer stemness. These findings 
provide a new direction for the diagnosis and treatment of HCC. In the future, if G1-G3 patients were treated with targeted therapy for PD-1/PD-L1 and liver cancer stem cells, this may improve the survival and prognosis of a patient with highly malignant HCC with an extremely poor prognosis.

The pathogenesis of HCC is complex and involves multiple signaling pathways[29,30]. Signaling pathways involved in growth factors activated by intracellular calcium ions play a crucial role in various physiological and biochemical processes[31]. HCC encodes a variety of tyrosine kinases, including epidermal growth factor receptor (EGFR), whose functions are mainly in glucose and lipid metabolism, liver fibrosis, regeneration, and carcinogenesis[32,33]. Modica et al.[34] reported that EGF-dependent HCC proliferation may be affected by calcium ion, and intracellular dissociative calcium is a key regulator for EGFR signal propagation and apoptosis of tumor cells. Calcium not only promotes the proliferation of proliferating HCC cells but also induces the apoptosis of non-proliferating HCC cells, which might contribute to the reason why the proliferation group (G1-G3) has a poor prognosis. Considering the high hepatic activity of calcium and the overexpression of calcium channels in G1-G3 group, manipulation of calcium influx in HCC cells may inhibit tumor metastasis.

ITPR1 encodes an intracellular receptor for type 1 inositol 1,4,5-triphosphate and mediates the release of calcium from the endoplasmic reticulum. A few recent studies indicated that ITPR1 affects tumor progression by regulating autophagy. For example, ITPR1 can protect renal cancer cells against natural killer cells by inducing autophagy[35,36]. In our study, we found that HCC patients with a high expression level of ITPR1 have a significantly lower 3-year OS rate than HCC patients with low or medium expression levels of ITPR1. Therefore, we speculate that ITPR1 may cooperate with calcium ions to promote the proliferation of proliferating HCC cells, thus leading to the increase of postoperative recurrence risk of HCC patients.

As a second messenger of cell information transmission, cGMP promotes cell division and inhibits cell differentiation. Our RNA-seq analysis showed that the cGMP-PKG signaling pathway was enriched in G1G3 type HCC, which might contribute to the proliferative characteristics of the G1-G3 subgroups. In this study, we found that G1-G3 type HCC patients showed a high serum AFP level. A differential analysis based on the HCC transcriptome data from TCGA showed that the genes expressed in HCC tissues with high AFP expression are involved in neuroactive ligand-receptor interaction pathways[37]. Together, these results suggest that the proliferative characteristics of G1-G3 subgroups might be from the enrichment of neuroactive ligand-receptor interaction pathways.

\section{Conclusions}

In this study, we found that the G1-G6 transcriptomic molecular classification was applicable in the China HCC cohort regardless of the ethnic origin of patients. Through the results of these cohorts, we may be able to assertively establish the clinical and pathological relevance of the 16 gene score and use the classification system to develop therapeutic strategies for HCC patients worldwide in the future. Our results help clarify our understanding of the correlation between G1-G6 molecular classification and 
molecular markers and molecular signaling pathways in the Chinese HCC population and initially established a link between the phenotype and molecular characteristics. However, we mostly performed correlation analysis of $\mathrm{G} 1-\mathrm{G} 6$ molecular classification, focusing on the postoperative prognosis monitoring of HCC patients, and more studies are needed to fully clarify the molecular mechanism of HCC occurrence and development.

\section{Abbreviations}

HCC, hepatocellular carcinoma; HBV, hepatitis B virus; HCV, hepatitis C virus; AFP, alpha-fetoprotein; REG1B, regenerating family member 1 beta; REG3G, regenerating family member 3 gamma; ITPR1, inositol 1,4,5-trisphosphate receptor type 1; MVI, microvascular invasion; HE, hematoxylin-eosin; TMA, Tissue microarray; IHC, immunohistochemistry; PD-L1, programmed death ligand 1

\section{Declarations}

\section{Acknowledgements}

The authors would like to thank Liyun Huang (Department of Pathology, Mengchao Hepatobiliary Hospital of Fujian Medical University, Fuzhou, Fujian, China) for her technical assistances.

\section{Authors' contributions}

HAM, CLH and HWB conceived and designed the experiments. CLH, ZZX and CRN performed the experiments. CLH, ZZX, CY and CRN analyzed the data. CLH, ZZX, HWB and CRN. wrote the paper. All authors read and approved the final manuscript.

\section{Funding}

This study was supported by Natural Science Foundation of Fujian Province (Fujian Provincial Natural Science Foundation): (Grant number: 2020J01605, 2020J011076); Fujian Medical Innovation Project: 2020CXA007; Startup Found for scientific research of Fujian Medical University (Grant number: 2019QH1295) and Sponsored by Fuzhou health technology project (2021-S-wq27).

\section{Availability of data and materials}

All data supporting the conclusions of this article are included in the manuscript files.

\section{Ethics approval and consent to participate}

The use of human clinical samples was approved by the Medical Ethics Committee of Mengchao Hepatobiliary Hospital of Fujian Medical University.

\section{Consent for publication}


All co-authors have seen and agree with the contents of the manuscript. We certify that the submission is an original work and is not under review by any other publication.

\section{Competing interests}

There is no financial interest to report.

\section{Authors' information}

1 Department of Pathology, The School of Basic Medical Sciences, Fujian Medical University, Fuzhou, Fujian, China

2 Institute of Oncology, Fujian Medical University, Fuzhou, Fujian, China

3 Diagnostic Pathology Center, Fujian Medical University, Fuzhou, Fujian, China

4 Department of Emergency, Fujian Provincial Hospital; Shengli Clinical Medical College of Fujian Medical University, Fuzhou, Fujian, China

5 Department of Pathology, Mengchao Hepatobiliary Hospital of Fujian Medical University, Fuzhou, Fujian, China

\section{References}

1. Chen W, Zheng R, Baade PD, Zhang S, Zeng H, Bray F, Jemal A, Yu XQ, He J. Cancer statistics in China, 2015. CA Cancer J Clin. 2016; 66 (2): 115-32.

2. Wang FS, Fan JG, Zhang Z, Gao B, Wang HY. The global burden of liver disease: the major impact of China. Hepatology. 2014; 60 (6): 2099-108.

3. Wang X, Mao J, Zhou T, Chen X, Tu H, Ma J, Li Y, Ding Y, Yang Y, Wu H, Tang X. Hypoxia-induced myeloid derived growth factor promotes hepatocellular carcinoma progression through remodeling tumor microenvironment. Theranostics. 2021; 11 (1): 209-21.

4. El-Serag HB, Rudolph KL. Hepatocellular carcinoma: epidemiology and molecular carcinogenesis. Gastroenterology. 2007; 132 (7): 2557-276.

5. Montella L, Palmieri G, Addeo R, Del Prete S. Hepatocellular carcinoma: Will novel targeted drugs really impact the next future? World J Gastroenterol. 2016; 22 (27): 6114-26.

6. Ahn S-M, Jang SJ, Shim JH, Kim D, Hong S-M, Sung CO, Baek D, Haq F. Genomic portrait of resectable hepatocellular carcinomas: implications of RB1 and FGF19 aberrations for patient stratification. Hepatology. 2014; 60 (6): 1972-82. 
7. Dong H, Qian Z, Zhang L, Chen Y, Ren Z, Ji Q. Genomic and transcriptome profiling identified both human and HBV genetic variations and their interactions in Chinese hepatocellular carcinoma. Genom Data. 2015; 6: 1-3.

8. Boyault S, Rickman DS, de Reyniès A, Balabaud C, Rebouissou S, Jeannot E, Hérault A. Transcriptome classification of HCC is related to gene alterations and to new therapeutic targets. Hepatology. 2007; 45 (1): $42-52$.

9. Allen JC, Nault JC, Zhu G, Khor AYK, Liu J, Lim TKH, Zucman-Rossi J, Chow PKH. The transcriptomic G1-G6 signature of hepatocellular carcinoma in an Asian population: association of G3 with microvascular invasion. Medicine (Baltimore). 2016; 95 (47): e5263.

10. Ahn SM, Haq F, Park I, Nault JC, Zucman-Rossi J, Yu E. The clinical implications of G1-G6 transcriptomic signature and 5-gene score in Korean patients with hepatocellular carcinoma. BMC Cancer. 2018; 18 (1): 571-77.

11. Woo HG, Wang XW, Budhu A, Kim YH, Kwon SM, Tang Z-Y, Sun Z, Harris CC, Thorgeirsson SS. Association of TP53 mutations with stem cell-like gene expression and survival of patients with hepatocellular carcinoma. Gastroenterology. 2011; 140 (3): 1063-70.

12. Schulze K, Imbeaud S, Letouzé E, Alexandrov LB, Calderaro J, Rebouissou S, Couchy G, Meiller C, Shinde J, Soysouvanh F, Calatayud AL. Exome sequencing of hepatocellular carcinomas identifies new mutational signatures and potential therapeutic targets. Nat Genet. 2015; 47 (5): 505-11.

13. Zhou Z, Qi J, Lim CW, Kim J-W, Kim B. Dual TBK1/IKKE inhibitor amlexanox mitigates palmitic acidinduced hepatotoxicity and lipoapoptosis in vitro. Toxicology. 2020; 444: 152579-594.

14. Zhou Z, Kim J-W, Qi J, Eo SK, Lim CW, Kim B. Toll-Like Receptor 5 Signaling Ameliorates Liver Fibrosis by Inducing Interferon B-Modulated IL-1 Receptor Antagonist in Mice. Am J Pathol. 2020; 190 (3): 614-29.

15. Rebouissou S, Nault JC. Advances in molecular classification and precision oncology in hepatocellular carcinoma. J Hepatol. 2020; 72 (2): 215-29.

16. Calderaro J, Ziol M, Paradis V, Zucman-Rossi J. Molecular and histological correlations in liver cancer. J Hepatol. 2019; 71 (3): 616-30.

17. Kwon JH, Lee N, Park JY, Yu YS, Kim JP, Shin JH, Kim DS, Joh JW, Kim DS. Actionable gene expression-based patient stratification for molecular targeted therapy in hepatocellular carcinoma. PLoS One. 2013; 8 (6): e64260.

18. El-Khoueiry AB, Sangro B, Yau T, Crocenzi TS, Kudo M, Hsu C, Kim T-Y, Choo S-P, Trojan J, Welling TH, Meyer T, Kang Y-K. Nivolumab in patients with advanced hepatocellular carcinoma (CheckMate 040): an open-label, non-comparative, phase 1/2 dose escalation and expansion trial. Lancet. 2017; 389 (10088): 2492-502. 
19. Calderaro J, Rousseau B, Amaddeo G, Mercey M, Charpy C, Costentin C, Luciani A, Zafrani E-S, Laurent A, Azoulay D, Lafdil F, Pawlotsky J-M. Programmed death ligand 1 expression in hepatocellular carcinoma: relationship with clinical and pathological features. Hepatology. 2016; 64 (6): 2038-46.

20. Calderaro J, Couchy G, Imbeaud S, Amaddeo G, Letouzé E, Blanc J-F, Laurent C, Hajji Y, Azoulay D, Bioulac-Sage P, Nault J-C, Zucman-Rossi J. Histological subtypes of hepatocellular carcinoma are related to gene mutations and molecular tumour classification. J Hepatol. 2017; 67 (4): 727-38.

21. Liu CQ, Xu J, Zhou ZG, Jin LL, Yu XJ, Xiao G, Lin J, Zhuang SM, Zhang YJ, Zheng L. Expression patterns of programmed death ligand 1 correlate with different microenvironments and patient prognosis in hepatocellular carcinoma. Br J Cancer. 2018; 119 (1): 80-88.

22. Wang W, Xu L, Liu P, Jairam K, Yin Y, Chen K, Sprengers D, Peppelenbosch MP, Pan Q, Smits R. Blocking Wnt Secretion Reduces Growth of Hepatocellular Carcinoma Cell Lines Mostly Independent of $\beta$ Catenin Signaling. Neoplasia. 2016; 18 (12): 711-23.

23. Shi W, Tang Y, Zhi Y, Li Z, Yu S, Jiang J, Zhu J, Li J, Wang F, Su L, Zhao X. Akt inhibition-dependent downregulation of the Wnt/beta-catenin signaling pathway contributes to antimony-induced neurotoxicity. Sci Total Environ. 2020; 737: 140252-259.

24. Malta TM, Sokolov A, Gentles AJ, Burzykowski T, Poisson L, Weinstein JN, Kamińska B, Huelsken J, Omberg L, Gevaert O, Colaprico A. Machine learning identifies stemness features associated with oncogenic dedifferentiation. Cell. 2018; 173 (2): 338-54.e15.

25. Miranda A, Hamilton PT, Zhang AW, Pattnaik S, Becht E, Mezheyeuski A, Bruun J, Micke P, de Reynies A, Nelson BH. Cancer stemness, intratumoral heterogeneity, and immune response across cancers. Proc Natl Acad Sci U S A. 2019; 116 (18): 9020-29.

26. Audard V, Grimber G, Elie C, Radenen B, Audebourg A, Letourneur F, Soubrane O, Vacher-Lavenu M-C, Perret C, Cavard C, Terris B. Cholestasis is a marker for hepatocellular carcinomas displaying beta-catenin mutations. J Pathol. 2007; 212 (3): 345-52.

27. Monga SP. $\beta$-catenin signaling and roles in liver homeostasis, injury, and tumorigenesis. Gastroenterology. 2015; 148 (7): 1294-310.

28. Spencer HL, Eastham AM, Merry CLR, Southgate TD, Perez-Campo F, Soncin F, Ritson S, Kemler R, Stern PL, Ward CM. E-cadherin inhibits cell surface localization of the pro-migratory 5T4 oncofetal antigen in mouse embryonic stem cells. Mol Biol Cell. 2007; 18 (8): 2838-51.

29. Villanueva A, Newell P, Chiang DY, Friedman SL, Llovet JM. Genomics and signaling pathways in hepatocellular carcinoma. Semin Liver Dis. 2007; 27 (1): 55-76.

30. Whittaker S, Marais R, Zhu AX. The role of signaling pathways in the development and treatment of hepatocellular carcinoma. Oncogene. 2010; 29 (36): 4989-5005. 
31. Berridge MJ, Lipp P, Bootman MD. The versatility and universality of calcium signalling. Nat Rev Mol Cell Biol. 2000; 1 (1): 11-21.

32. Trusolino L, Bertotti A, Comoglio PM. MET signalling: principles and functions in development, organ regeneration and cancer. Nat Rev Mol Cell Biol. 2010; 11 (12): 834-48.

33. Joffre C, Barrow R, Ménard L, Calleja V, Hart IR, Kermorgant S. A direct role for Met endocytosis in tumorigenesis. Nat Cell Biol. 2011; 13 (7): 827-37.

34. Modica TME, Dituri F, Mancarella S, Pisano C, Fabregat I, Giannelli G. Calcium regulates HCC proliferation as well as EGFR recycling/degradation and could be a new therapeutic target in HCC. Cancers (Basel). 2019; 11 (10): 1588-606.

35. Messai Y, Noman MZ, Hasmim M, Janji B, Tittarelli A, Boutet M, Baud V, Viry E, Billot K, Nanbakhsh A, Ben Safta T, Richon C. ITPR1 protects renal cancer cells against natural killer cells by inducing autophagy. Cancer Res. 2014; 74 (23): 6820-32.

36. Xu S, Wang P, Zhang J, Wu H, Sui S, Zhang J, Wang Q, Qiao K, Yang W, Xu H, Pang D. Ai-IncRNA EGOT enhancing autophagy sensitizes paclitaxel cytotoxicity via upregulation of ITPR1 expression by RNA-RNA and RNA-protein interactions in human cancer. Mol Cancer. 2019; 18 (1): 89-106.

37. Wang XJ, Shen RF, Wang X, Wang YR, Xiao T. Differential analysis of gene expression profiles in hepatocellular carcinoma patients with high and low levels of alpha-fetoprotein. Zhonghua Zhong Liu Za Zhi. 2020; 42 (5): 396-402.

\section{Figures}


China cohort $(n=107)$

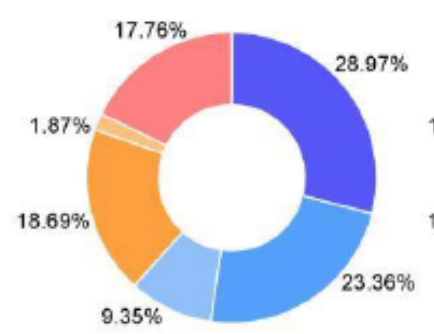

Europe cohort $(n=120)$

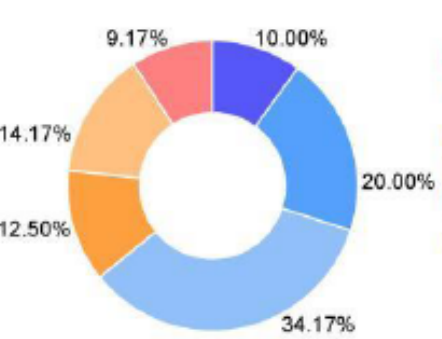

.

\section{C}

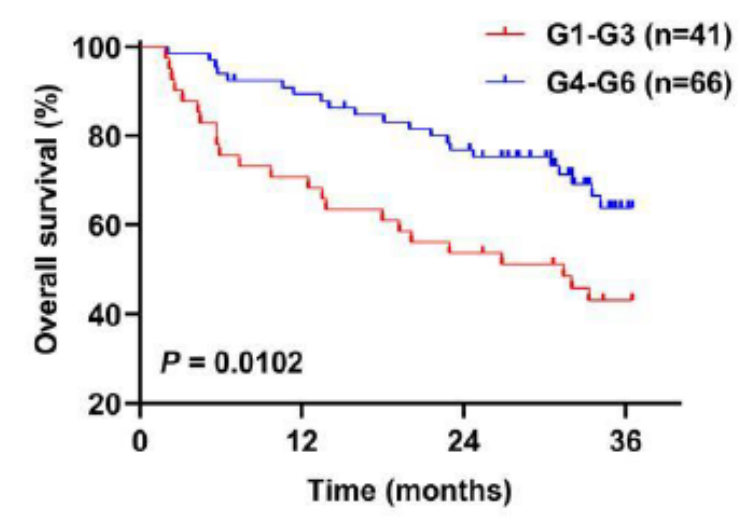

E

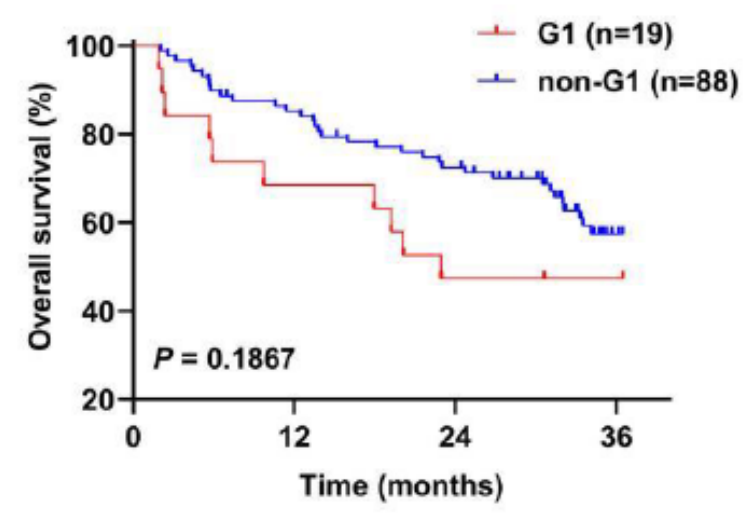$$
\text { ( }
$$

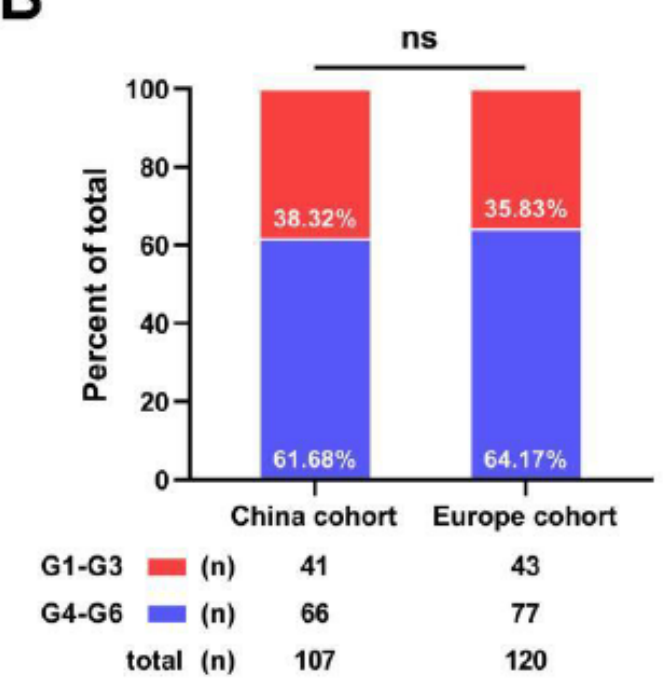

D

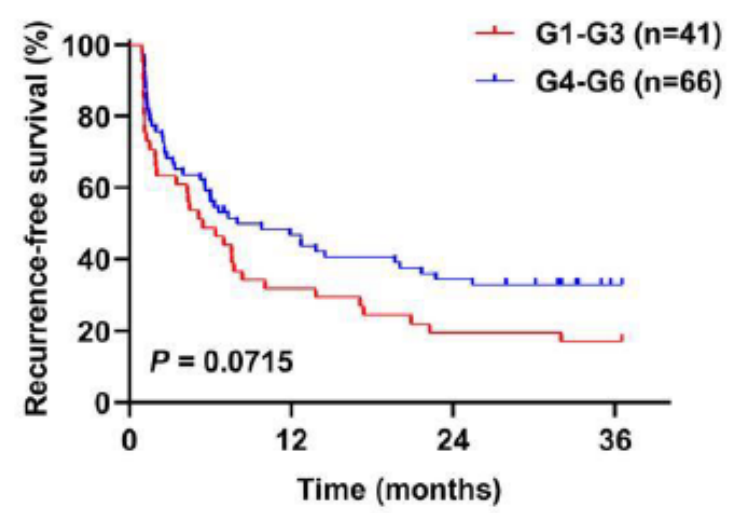

$\mathbf{F}$

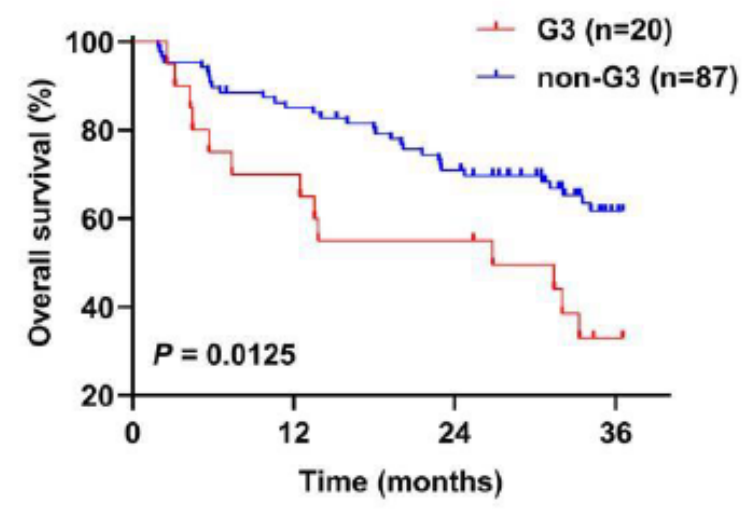

\section{Figure 1}

Distribution of G1-G6 subgroups in HCC patients from Europe and China studies (A) Distribution of HCC cohorts in China and Europe, $P<0.0001$; (B) distribution of the proliferative group ( $G 1-G 3, n=41)$ and nonproliferative group (G4-G6, $n=66$ ) in the HCC cohorts in China and Europe, $P=0.783$. The statistical difference between the groups was determined by Fisher exact probability method. (C) The 3-year OS rate was analyzed in the G1-G3 group and G4-G6 group; the statistical difference between the two groups 
was determined by Kaplan-Meier method, $P=0.0102$. (D) The recurrence-free survival of the $\mathrm{G} 1-\mathrm{G} 3$ group $(n=41)$ and $G 4-G 6$ group $(n=66)$ was determined by Kaplan-Meier method, $P=0.0715$. (E and $F)$ The difference in 3-year OS between the G1 or G3 group and other groups was analyzed by Fisher exact probability method.
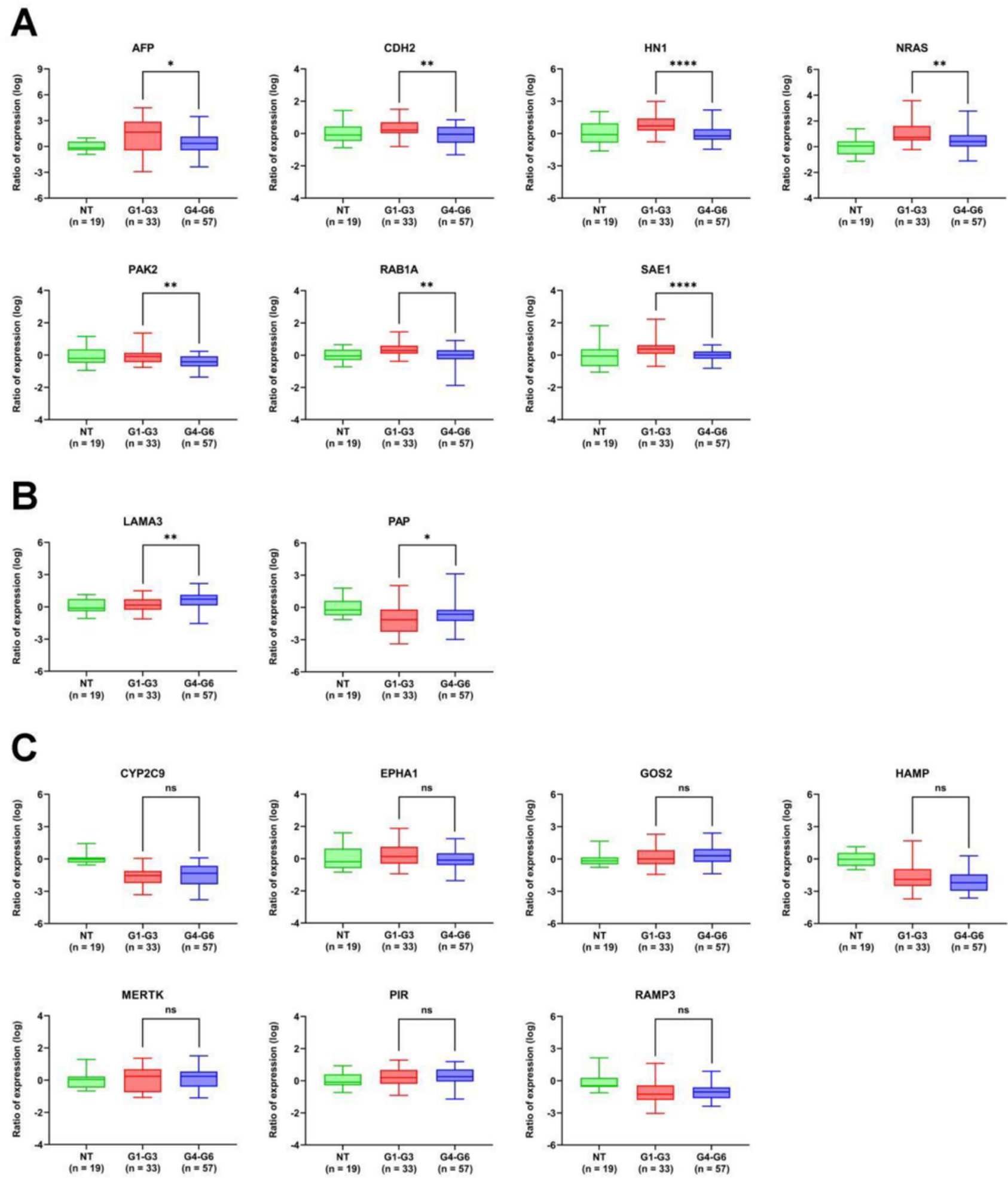

Figure 2 
Predictor of HCC classification using qPCR qPCR was used to evaluate the expression of the 16 genes in $90 \mathrm{HCC}$ samples and 19 randomly selected para-cancer liver tissue samples. (A) qPCR results for AFP, $\mathrm{CDH} 2$, HN1, NRAS, PAK2, RAB1A, and SAE1. (B) qPCR results for LAMA3 and PAP. (C) qPCR results for CYP2C9, EPHA1, G0S2, HAMP, MERTK, PIR, and RAMP3. Data are presented as mean \pm standard deviation. ${ }^{*} P<0.05, * * P<0.01,{ }^{* * *} P<0.001,{ }^{* * * *} P<0.0001 ; n$, no statistical difference.

A
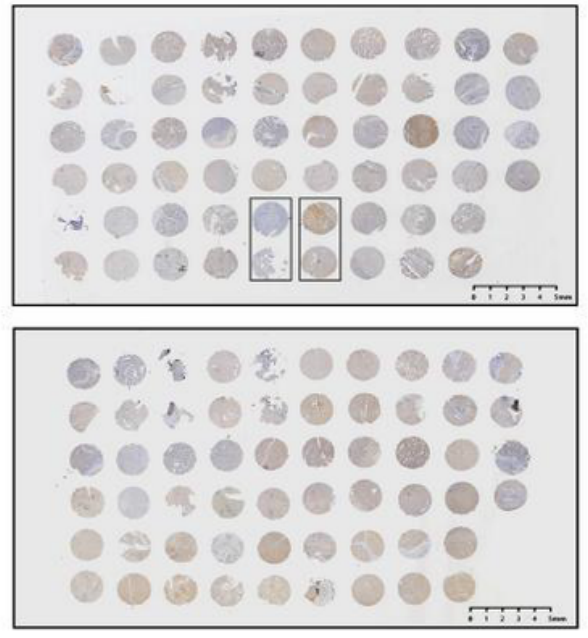

B
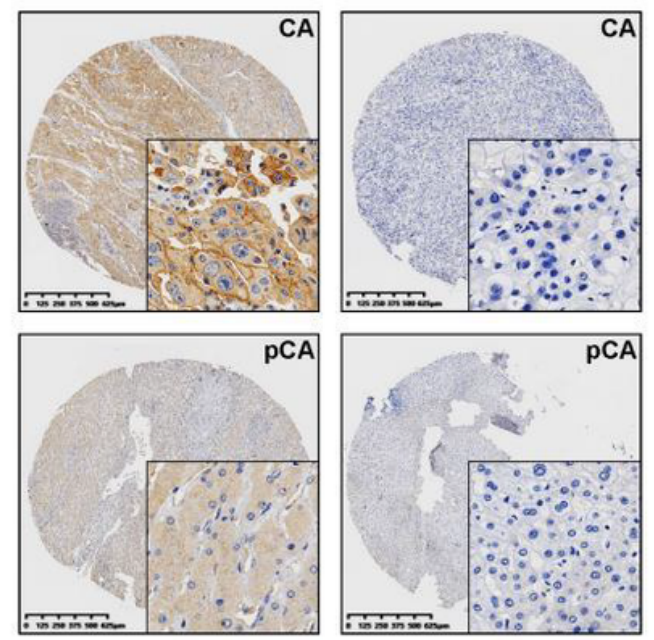

C

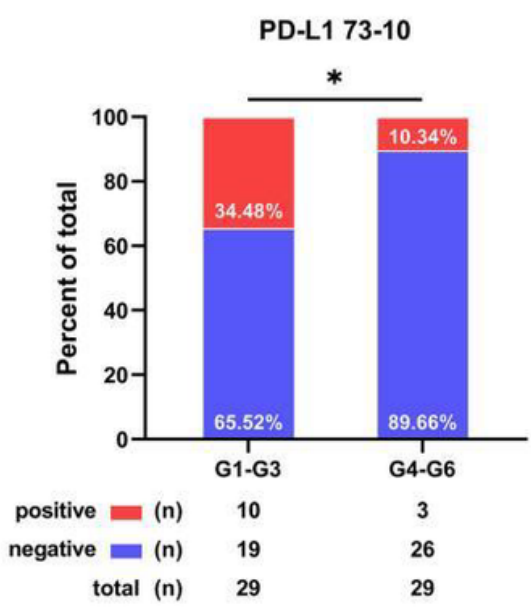

Figure 3

PD-L1 is highly expressed in G1-G3 subgroups (A) The arrangement of the TMA. (B) Representative images for the positive area of PD-L1 antibody. (C) The percentage of positive PD-L1 staining was analyzed. The difference was detected by the chi-square test. ${ }^{*} P<0.05$. 
A
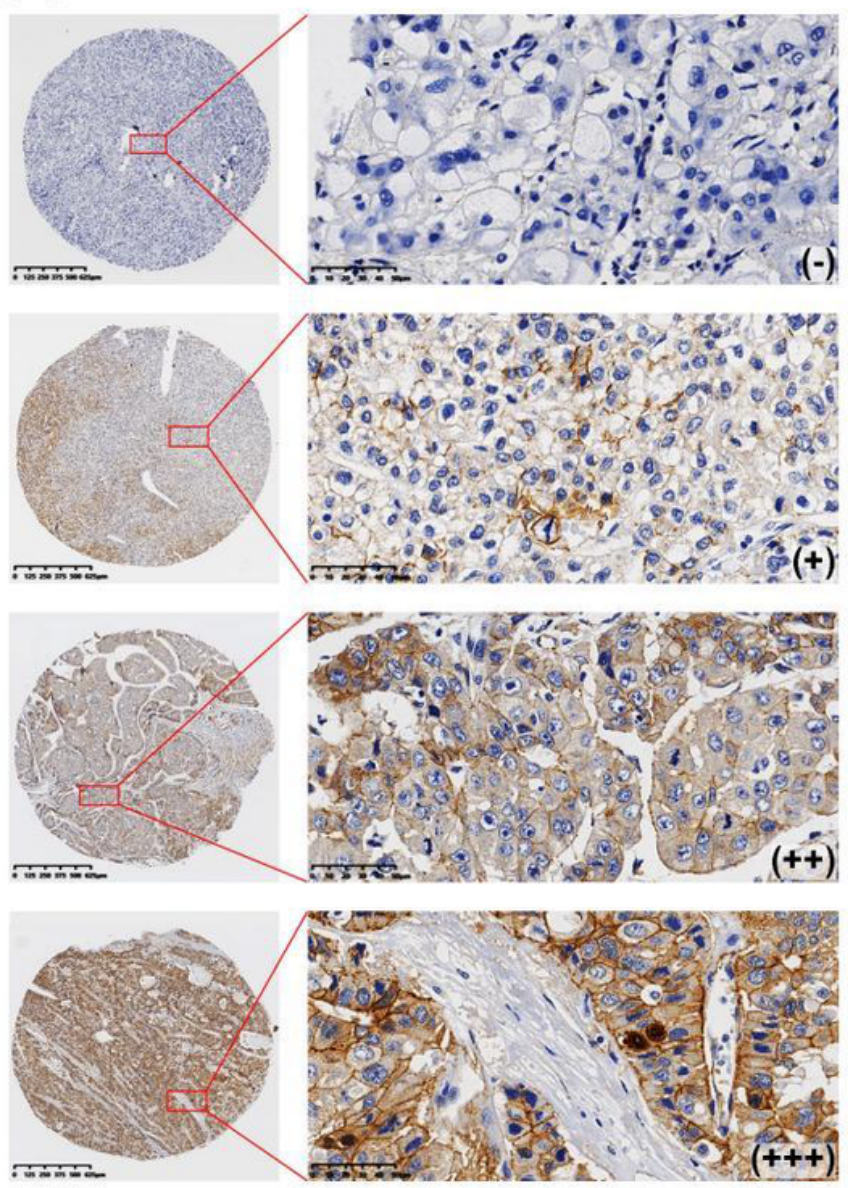

D
100KD

$36 \mathrm{KD}$

GS

$---\infty$

GAPDH

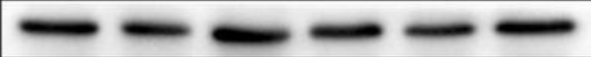

B

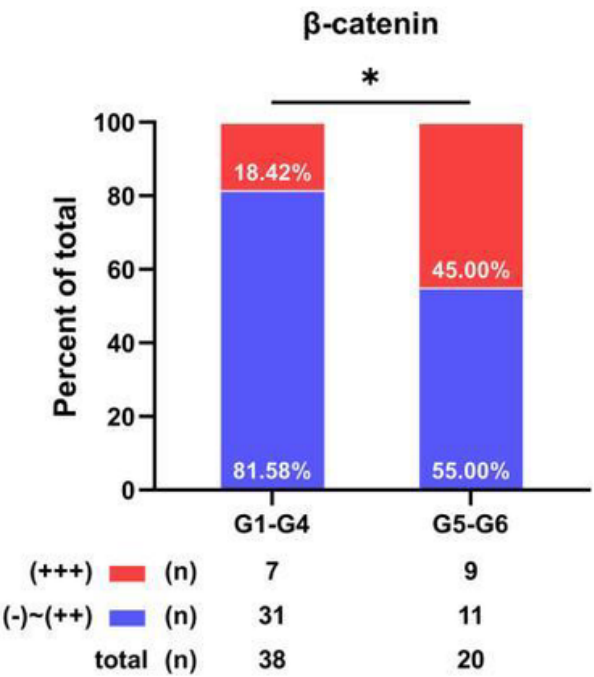

C

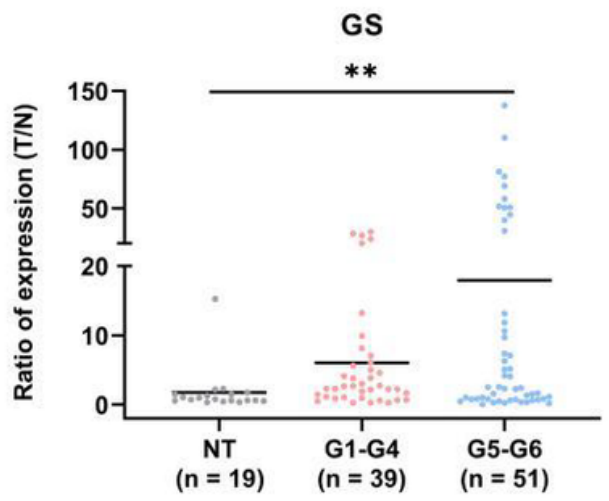

E
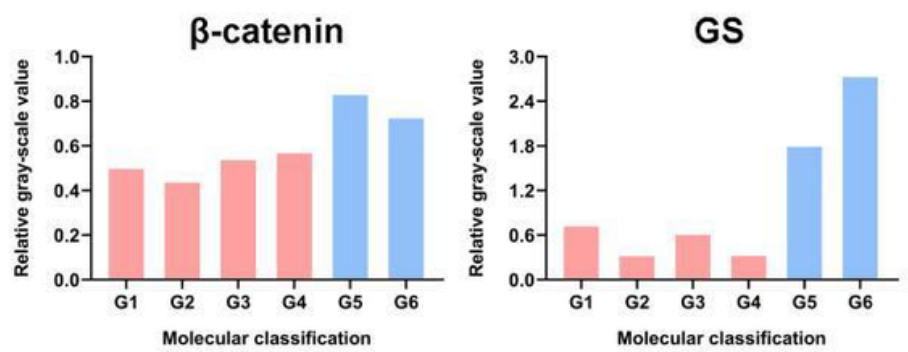

Figure 4

G5 and G6 HCC subgroups are associated with activation of the Wnt/ $\beta$-catenin pathway signaling The expression of $\beta$-catenin and GS were evaluated. (A) Representative images for the positive $\beta$-catenin staining in $58 \mathrm{HCC}$ tissues. (B) Percentages of positive $\beta$-catenin expression in G1-G4 and G5-G6 groups. (C) The expression of GS mRNA in $90 \mathrm{HCC}$ samples and 19 para-cancer liver tissue samples was determined by qPCR. ( $D$ and $E$ ) The protein levels of $\beta$-catenin and GS in 6 HCC samples were measured by western blot. ${ }^{*} \mathrm{P}<0.05,{ }^{*} \mathrm{P}<0.01$. 
A

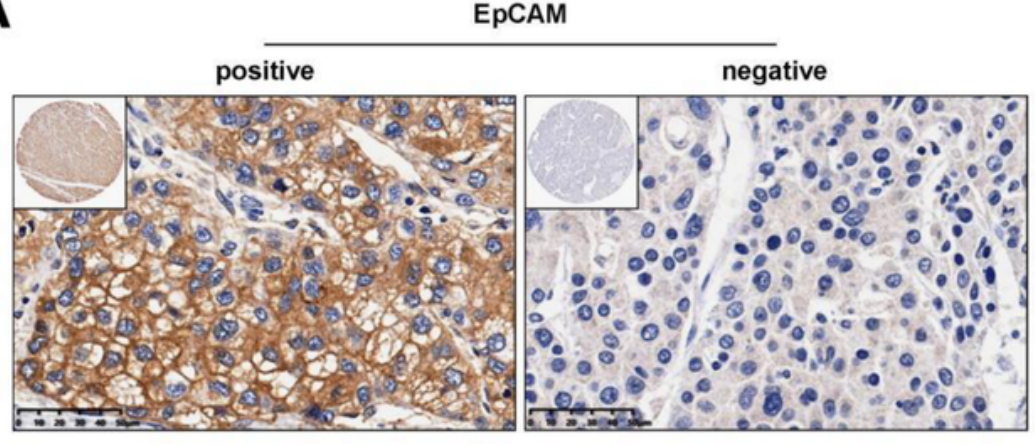

C

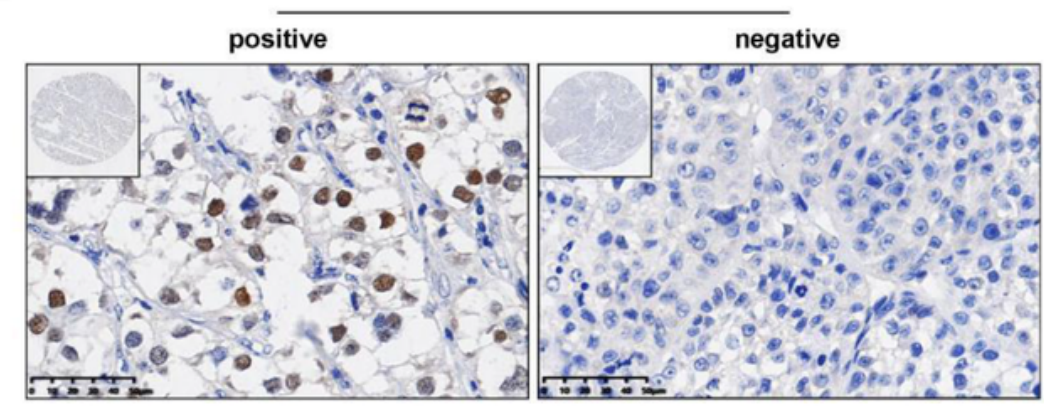

B

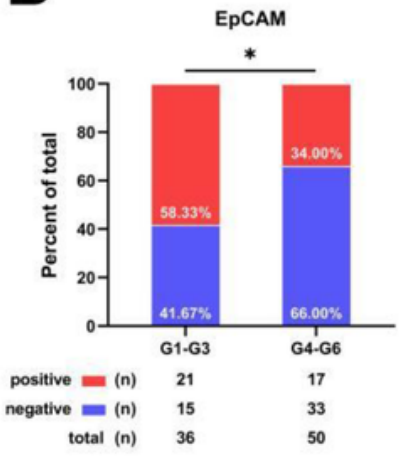

D

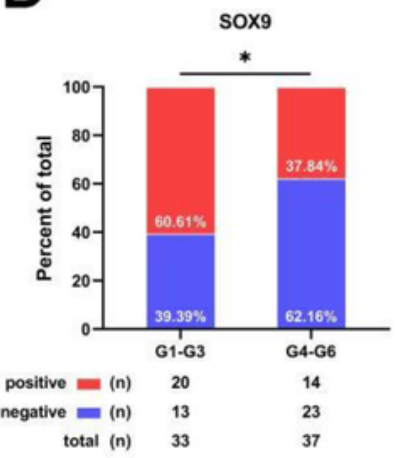

$\mathbf{E}$

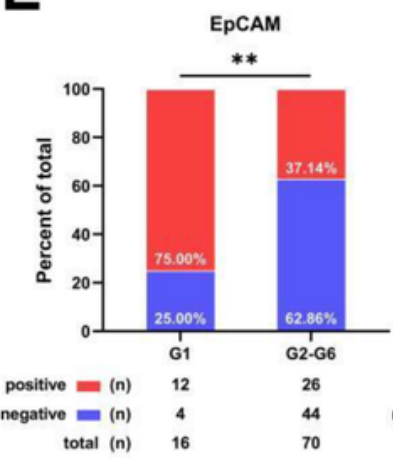

G

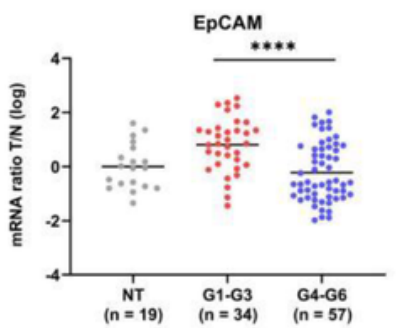

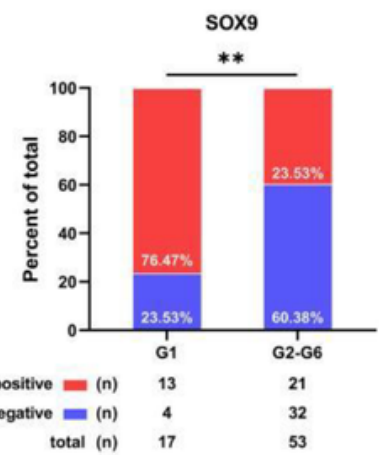

total $(n)$
$\mathbf{F}$
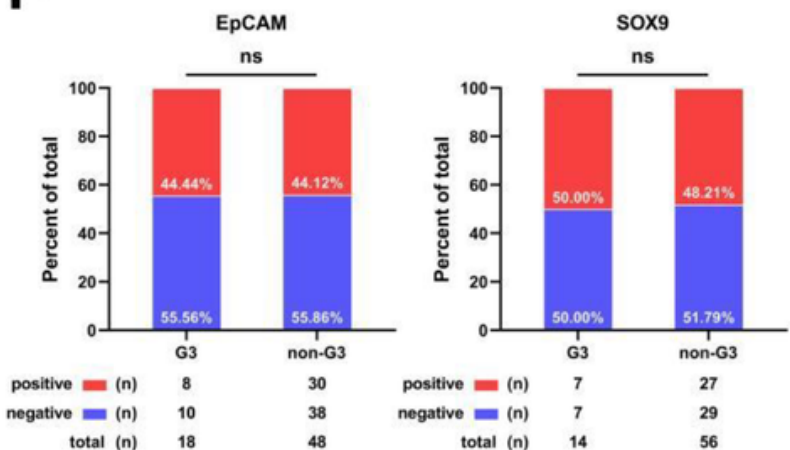

H
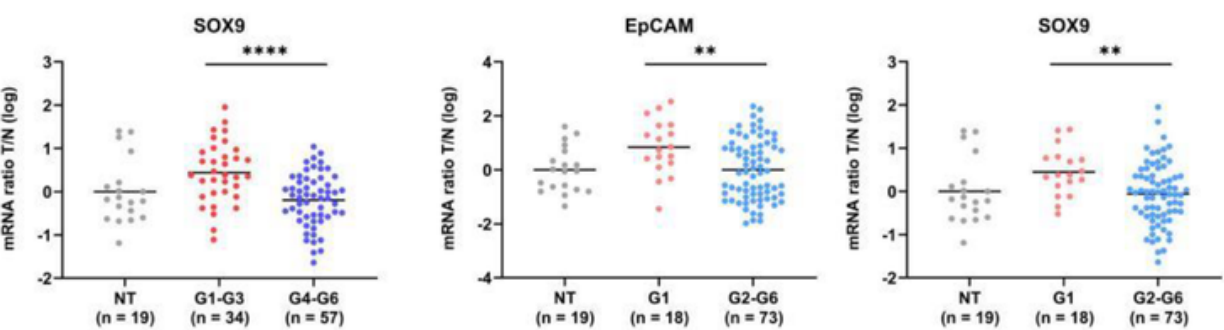

\section{Figure 5}

The $\mathrm{G} 1$ subgroup is associated with maintenance of stemness in tumor cells ( $A$ and $B$ ) Representative images for the positive EpCAM staining and the positive percentages of EpCAM in the G1-G3 group and G4-G6 group. (C and D) Representative images for positive SOX9 staining and percentages. (E) The percentages of positive EpCAM and SOX9 in the G1 group and G2-G6 group. (F) The percentages of positive EpCAM and SOX9 in the G3 subgroup and other subgroups. (G) The expressions of EpCAM and 
SOX9 mRNA in the G1-G3 group and G4-G6 group were determined by qPCR. $(H)$ The expressions of EpCAM and SOX9 mRNA in the G1 subgroup and G2-G6 subgroups were determined by qPCR. ${ }^{*} P<0.01$, $* * * P<0.001, * * * * P<0.0001 ;$ ns, no statistical difference.
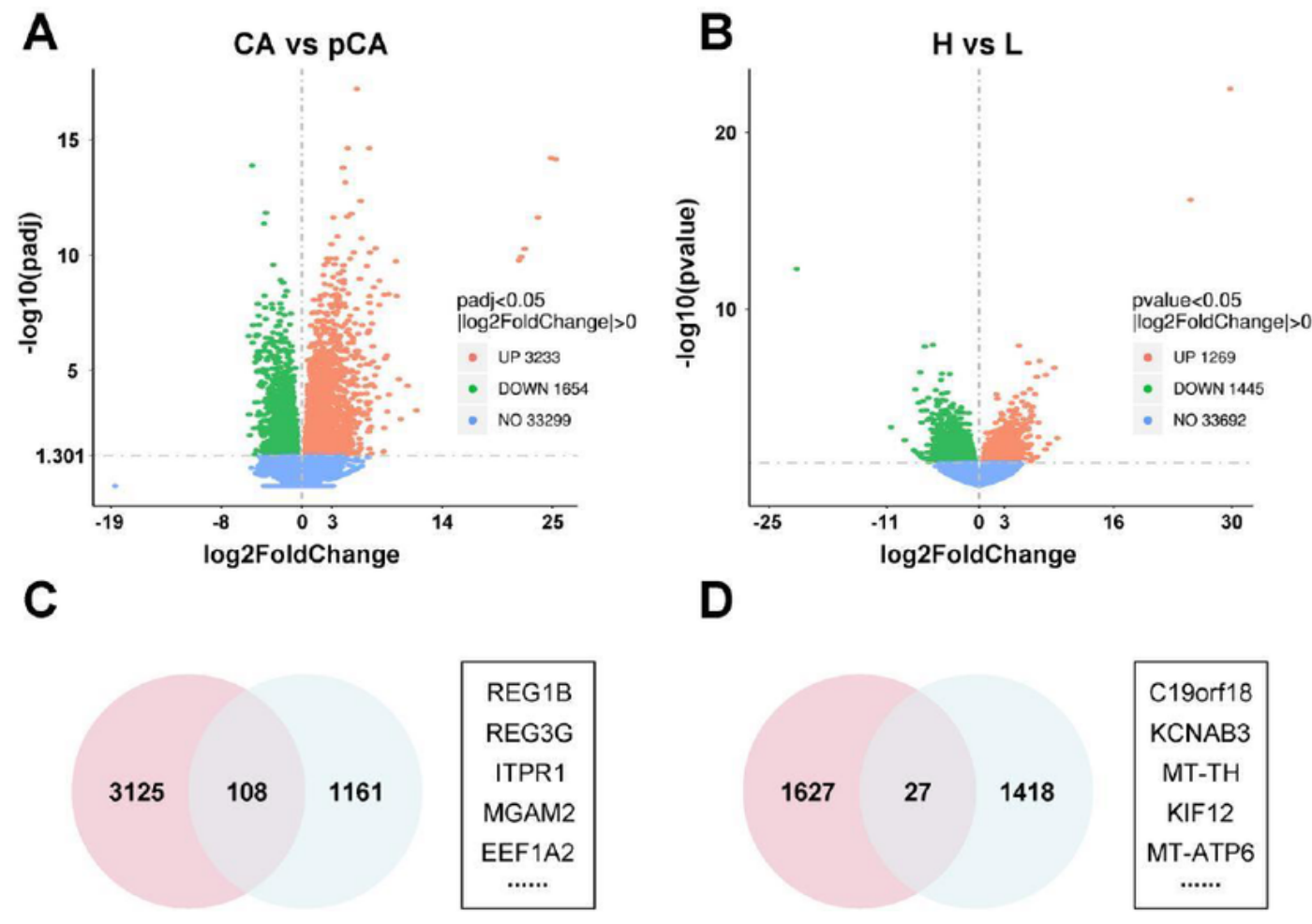

D

CA vs pCA - up H vs L - up

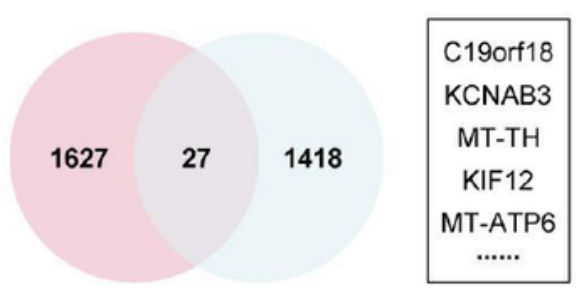

CA vs pCA - down H vs L - down
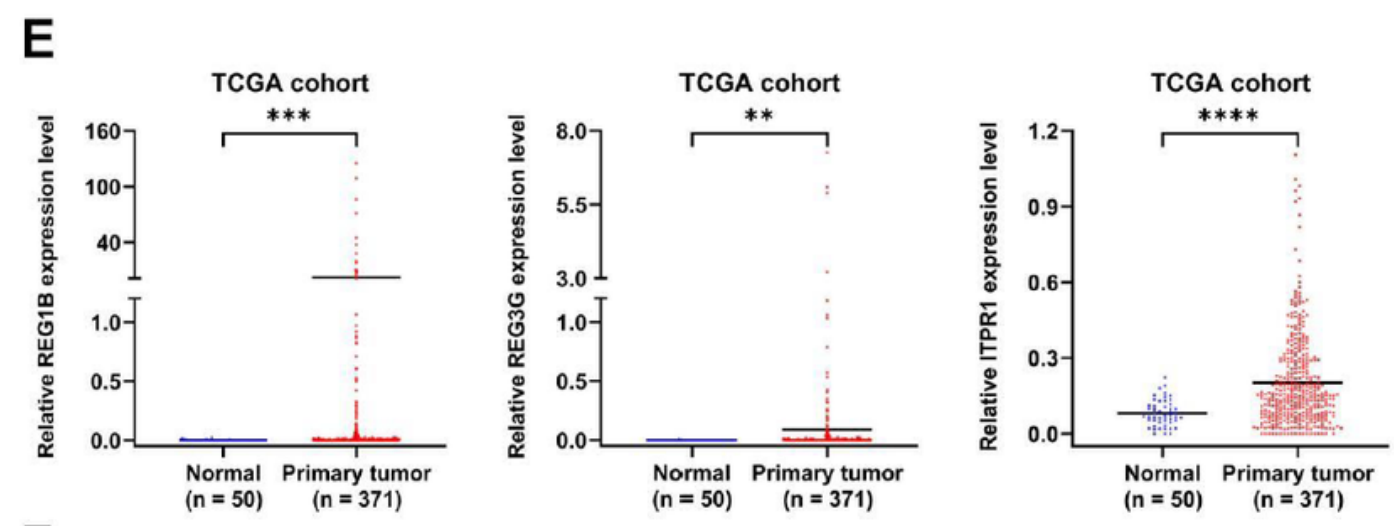

$\mathbf{F}$
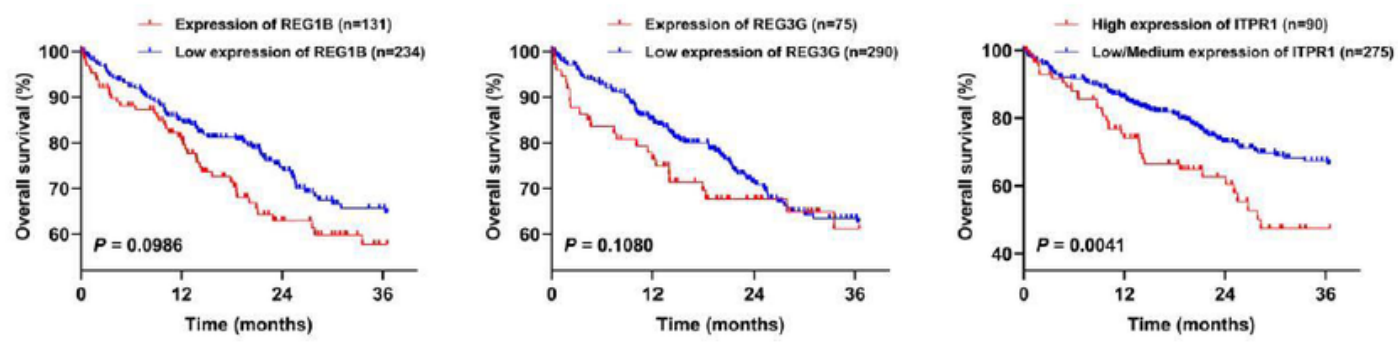

Figure 6

Transcriptome sequencing analysis for $\mathrm{G} 1-\mathrm{G} 6$ molecular classification of Chinese HCC patients (A) Volcano plot of differentially expressed genes between the CA and pCA samples $(n=12)$. (B) Volcano plot 
of differentially expressed genes between the $H$ and $L$ groups $(n=6)$. (C) Venn map of overlapped genes with upregulation. (D) Venn map of overlapped genes with downregulation. (E) TCGA data showed that the expression levels of REG1B, REG3G, and ITPR1 were higher in HCC tissues than in normal liver tissues. (F) Data analysis from TCGA database showed that patients with high expression of ITPR1 had a worse prognosis than those with moderate/low expression. ${ }^{* * P} P<0.01, * * * P<0.001$, and ${ }^{* \star * *} P<0.0001$.
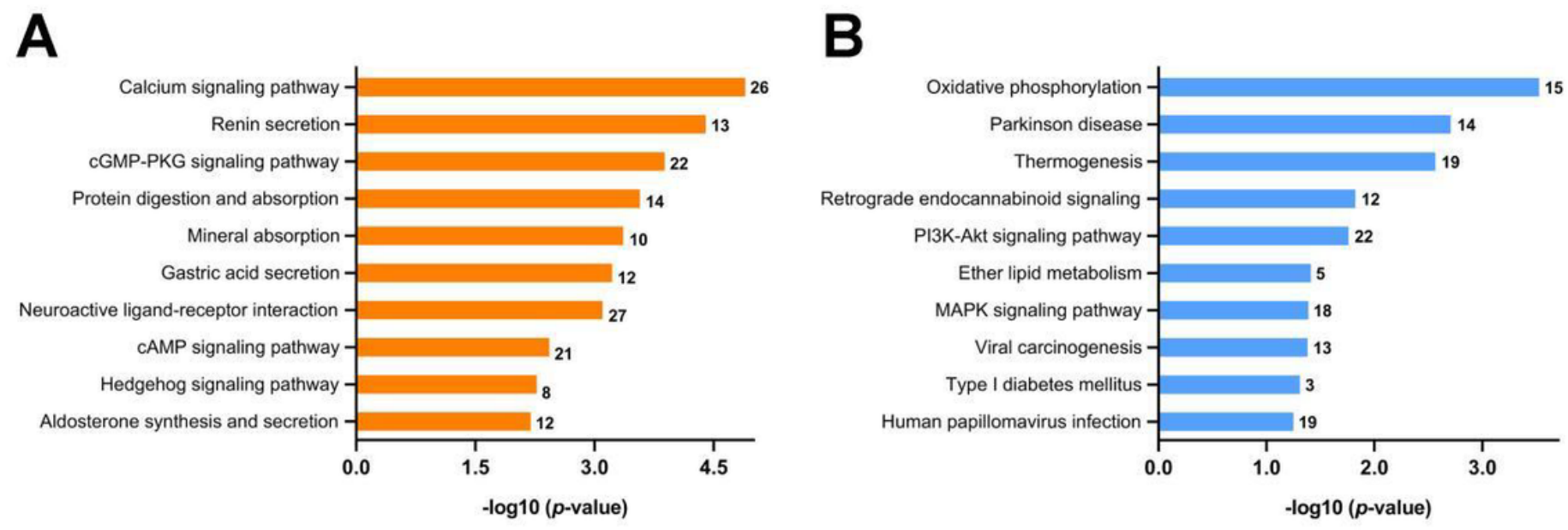

\section{Figure 7}

KEGG pathway enrichment analysis of differential gene expression (A) KEGG pathway enrichment analysis of upregulated differentially expressed genes. (B) KEGG pathway enrichment analysis of downregulated differentially expressed genes.

\section{Supplementary Files}

This is a list of supplementary files associated with this preprint. Click to download.

- SupplementaryFigure1.png

- SupplementaryFigure2.png 